THE ASTROPHySiCAL JOURNAL SUPPLEMENT SERIES, 96:303-324, 1995 January

(c) 1995. The American Astronomical Society. All rights reserved. Printed in U.S.A.

\title{
CALIBRATING THE BROAD BAND X-RAY TELESCOPE
}

\author{
K. A. Weaver, ${ }^{1,2}$ K. A. Arnaud,${ }^{1}$ E. A. Boldt, D. Christian, ${ }^{1}$ M. Corcoran, ${ }^{3}$ S. S. Holt, K. Jahoda, R. Kelley, \\ F. E. Marshall, R. F. Mushotzky, R. Petre, G. Rawley, ${ }^{4}$ P. J. Serlemitsos, E. M. Schlegel, ${ }^{3}$ \\ A. P. SMALE, ${ }^{3}$ J. H. SWANK, AND A. E. SZYMKOWIAK \\ Code 666, NASA/Goddard Space Flight Center, Greenbelt, MD 20771 \\ AND \\ S. YAMAUCHI \\ Institute of Physical and Chemical Research, 2-1 Hirosawa, Wako Saitama, 351-01, Japan \\ Received 1993 October 25; accepted 1994 June 29
}

\begin{abstract}
This paper describes the calibration of the two solid-state $\mathrm{Si}(\mathrm{Li}) \mathrm{X}$-ray detectors and the X-ray telescopes that flew as part of Goddard Space Flight Center's Broad Band X-Ray Telescope (BBXRT) experiment on board the space shuttle Columbia in 1990 December. During the 9 day shuttle mission, BBXRT performed $\sim 150$ observations of 82 celestial sources. The content of the archive is summarized here. Although BBXRT had a relatively short life, it stands as a milestone in X-ray astronomy as being the first instrument to offer moderate spectral resolution over a wide bandpass (0.3-12.0 keV). Among other things, this paper discusses the effective area calibration of the instrument, the flux calibration and flux corrections for off-axis observations, the detector background, and optimal background subtraction techniques. The on-axis effective area calibration for the central detector elements was performed using data from the Crab Nebula, while other carefully selected targets were used for calibration of the outer detector elements. The remaining systematic uncertainties in the effective area calibration for point sources observed both on and off axis are generally less than 5\%-10\%. The energy scale is known to better than $0.5 \%$ at $6.0 \mathrm{keV}$ for both detectors. The results presented here have an impact on the calibration of other medium resolution X-ray experiments such as the CCDs on board $A S C A(A s t r o-D)$ as well as the Advanced $X$-Ray Astrophysics Facility detectors.
\end{abstract}

Subject headings: instrumentation: detectors - space vehicles — X-rays: general

\section{INTRODUCTION}

The Broad Band X-Ray Telescope was flown in 1990 December on board the space shuttle Columbia (STS-35) as part of Astro-1, the first NASA shuttle mission devoted entirely to astronomical observations. BBXRT consisted of two identical telescopes with lightweight, nested, thin-foil conical imaging mirrors (Serlemitsos 1988), which focused X-rays onto two segmented, cryogenically cooled, spectrophotometric $\mathrm{Si}(\mathrm{Li})$ diodes located at the focal plane. The mirrors were invented, designed, and built at the Goddard Space Flight Center (GSFC) Laboratory for High Energy Astrophysics; the detectors were supplied by Lawrence Berkeley Laboratory. The only solid-state detector and mirror combination flown in orbit prior to BBXRT was the Solid-State Spectrometer (SSS) on the Einstein Observatory. BBXRT is modeled after the SSS; however, BBXRT has much better energy resolution $(10 \%$ compared to $20 \%$ at $0.8 \mathrm{keV}$ ), about 4 times the effective area at $1 \mathrm{keV}$, a factor of 3 larger bandpass, and a factor of 100 less internal background. Prior to the flight of BBXRT, the technique of X-ray spectroscopy was limited by either the poor energy resolution of proportional counter experiments or the

\footnotetext{
${ }^{1}$ Also University of Maryland, College Park.

${ }^{2}$ Present address: Department of Astronomy and Astrophysics, 525 Davey Lab, Pennsylvania State University, University Park, PA 16802.

${ }^{3}$ With the Universities Space Research Association.

${ }^{4}$ With the Applied Research Corporation.
}

small bandpass and low efficiency of instruments with higher spectral resolution.

The large bandpass (0.3-12.0 keV) and good energy resolution of BBXRT $(\sim 2.5 \%$ at $6 \mathrm{keV})$ combined with the extremely low detector background $\left(1.5 \times 10^{-3}\right.$ counts $\mathrm{s}^{-1}$ $\mathrm{keV}^{-1}$ in each central detector element) make the instrument ideal for studying complex spectra and searching for X-ray emission and absorption features. The objectives of the BBXRT mission were to perform X-ray spectroscopy in the $0.3-12.0 \mathrm{keV}$ band, to observe $\mathrm{X}$-ray spectral features with particular emphasis placed on iron $\mathrm{K}$ lines, and to study broadband spectral variability. During its flight, BBXRT spent roughly $185,000 \mathrm{~s}$ pointing at celestial sources with typical integration times of 500-2000 s per observation. The BBXRT observation log, containing a complete list of all scientifically useful pointings, is given in Table 1 . The targets observed include stars, X-ray binaries, supernova remnants, normal galaxies, active galaxies, and clusters of galaxies. Observations of the diffuse X-ray background, Earth's atmosphere, and the reflected solar spectrum are not listed in Table 1, but account for a large fraction of the total observing time.

This paper presents a detailed description of the calibration of the BBXRT experiment. The characteristics of the detector and mirrors are presented in $\S 2.1$ and $\S 2.2$, followed by a short description of the mission and the in-flight performance of $\operatorname{BBXRT}(\S 2.3)$. The detector gain, resolution, and incomplete charge collection are summarized in $\$ \S 3.1-3.3$. The Crab Neb- 
TABLE 1

BBXRT OBSERVATION LOG

\begin{tabular}{|c|c|c|c|c|c|c|c|c|c|c|c|c|}
\hline Source & R.A. & Decl. ${ }^{a}$ & R.A. offset ${ }^{\text {b }}$ & Decl. offset ${ }^{b}$ & Roll $^{c}$ & Start $^{d}$ & $\operatorname{Exp}^{e}$ & $\mathrm{D} / \mathrm{N}^{\mathrm{f}}$ & $\mathrm{GR}^{\mathrm{g}}$ & Angle $^{h}$ & Rate $^{\mathrm{i}}$ & Class ${ }^{j}$ \\
\hline GAM-CAS & 13.413 & 60.445 & -0.055 & -0.086 & 19.3 & 1.2061 & 233.3 & $\mathbf{N}$ & 1284.4 & 5.4 & 7.82 & MXRB \\
\hline CAPELLA & 78.248 & 45.950 & & & 40.0 & 2.2195 & 1947.2 & MN & 1218.2 & -1.0 & 2.75 & RSCVn \\
\hline CAPELLA & 78.248 & 45.950 & & & 39.0 & 2.2836 & 1706.1 & B & 1136.0 & -1.0 & 3.15 & RSCVn \\
\hline CRAB & 82.870 & 21.978 & & & 154.0 & 2.4907 & 112.3 & D & 948.0 & -1.0 & 1127.36 & SNR \\
\hline CRAB & 82.870 & 21.978 & 0.144 & 0.278 & 153.2 & 2.4935 & 440.6 & D & 943.0 & -1.0 & 1418.07 & SNR \\
\hline CYG X-3 & 307.657 & 40.787 & & & 241.0 & 2.7306 & 384.9 & $\mathbf{N}$ & 1136.1 & 2.7 & 240.63 & LMXB \\
\hline PERSEUS & 49.123 & 41.331 & 0.023 & -0.032 & 120.7 & 2.7828 & 478.1 & $\mathbf{N}$ & 1418.4 & 2.2 & 9.46 & Cluster \\
\hline LMC X-2 & 80.325 & -72.007 & & & 277.0 & 2.8329 & 31.1 & D & 1272.5 & -1.0 & 16.01 & LMXB \\
\hline LMC X-2 & 80.325 & -72.007 & & & 280.0 & 2.9765 & 43.2 & $\mathbf{N}$ & 1010.2 & -1.0 & 12.04 & LMXB \\
\hline A754 & 136.660 & -9.460 & 0.020 & -0.020 & 330.6 & 2.9991 & 1454.8 & D & 1432.5 & 1.6 & 6.64 & Cluster \\
\hline CRAB & 82.870 & 21.978 & 0.050 & -0.001 & 354.1 & 3.0403 & 1270.1 & $N$ & 1599.9 & 3.0 & 1519.20 & SNR \\
\hline CRAB & 82.870 & 21.978 & & & 355.0 & 3.0550 & 1234.2 & B & 1367.9 & 1.0 & 1521.19 & SNR \\
\hline AR-LAC & 331.664 & 45.496 & -0.024 & -0.045 & 22.7 & 3.0834 & 1456.4 & D & 1327.6 & 2.9 & 4.80 & RSCVn \\
\hline M81 & 147.865 & 69.304 & & & 37.0 & 3.1053 & 2734.5 & MN & 1476.3 & -1.0 & 2.69 & Starburst \\
\hline CAS-A & 350.292 & 58.540 & & & 183.0 & 3.1493 & 996.2 & D & 1186.4 & -1.0 & 48.31 & SNR \\
\hline EZ-CMA & 103.034 & -23.864 & 0.062 & 0.129 & 256.7 & 3.1684 & 2522.0 & MD & 1216.3 & 8.5 & 0.90 & W-Rayet \\
\hline TON490 & 152.775 & 25.100 & 0.048 & -0.023 & 342.2 & 3.2510 & 1061.9 & D & 1152.6 & 2.9 & 1.07 & RL QSO \\
\hline СТВ109 & 345.000 & 58.633 & 0.292 & 0.122 & 186.8 & 3.2784 & 2237.4 & D & 1038.8 & 11.7 & 6.24 & SNR \\
\hline PUPAC & 125.625 & -42.800 & -0.118 & -0.031 & 317.7 & 3.3278 & 794.9 & D & 7317.6 & 5.5 & 33.83 & SNR \\
\hline $0614+09$ & 93.595 & 9.156 & 0.081 & -0.082 & 40.7 & 3.3598 & 915.2 & $\mathrm{~N}$ & 1023.7 & 6.8 & 54.03 & LMXB \\
\hline $0614+09$ & 93.595 & 9.156 & 0.011 & -0.007 & 39.1 & 3.3728 & 406.1 & $\mathrm{~N}$ & 1038.4 & 0.7 & 66.44 & LMXB \\
\hline $0748-676$ & 117.102 & -67.623 & 0.084 & 0.067 & 280.6 & 3.3831 & 1363.6 & D & 1087.3 & 4.5 & 22.26 & LMXB \\
\hline A496 & 67.835 & -13.363 & -0.034 & 0.047 & 29.2 & 3.4126 & 2048.4 & $N$ & 988.2 & 3.5 & 6.37 & Cluster \\
\hline $1634+706$ & 248.717 & 70.627 & & & 275.0 & 3.5768 & 2147.1 & D & 1122.4 & -1.0 & 10.70 & RQ QSO \\
\hline A2319 & 289.908 & 43.841 & & & 194.0 & 3.6500 & 388.8 & D & 1363.0 & -1.0 & 83.50 & Cluster \\
\hline CAPELLA & 78.248 & 45.950 & & & 215.0 & 3.7433 & 118.4 & $\mathrm{~N}$ & 1397.8 & -1.0 & 10.49 & RSCVn \\
\hline PERSEUS & 49.123 & 41.331 & & & 233.0 & 3.7570 & 199.7 & $\mathbf{N}$ & 952.5 & -1.0 & 47. & \\
\hline LMC X-1 & 85.023 & -69.768 & & & 280.0 & 3.7828 & 616.4 & D & 1151.8 & -1.0 & 32.72 & MXRB \\
\hline NGC2110 & 87.443 & -7.467 & -0.030 & 0.033 & 301.6 & 3.7994 & 2455.3 & $\mathrm{~N}$ & 1358.8 & 2.7 & 1.79 & Seyfert 2 \\
\hline NGC4151 & 182.004 & 39.684 & & & 330.0 & 3.8331 & 893.6 & D & 1853.5 & 5.5 & 7.04 & Seyfert 1.5 \\
\hline CAS-A & 350.292 & 58.540 & & & 205.0 & 3.8515 & 106.9 & $\mathbf{D}$ & 1012.9 & -1.0 & 137.77 & SNR \\
\hline MKN335 & 0.9380 & 19.925 & & & 205.0 & 3.8544 & 2081.1 & MN & 1470.4 & 2.0 & 2.83 & Seyfert 1 \\
\hline MKN335 & 0.9380 & 19.925 & & & 205.0 & 3.8800 & 232.2 & $\mathrm{~N}$ & 1102.7 & -1.0 & 2.33 & Seyfert 1 \\
\hline MKN876 & 243.401 & 65.844 & & & 273.0 & 3.9106 & 322.0 & D & 955.7 & 1.6 & 5.53 & Seyfert 1 \\
\hline Q1821 & 275.433 & 64.325 & & & 249.0 & 3.9425 & 388.8 & $\mathrm{~N}$ & 1164.4 & -1.0 & 2.67 & RQ QSO \\
\hline CYG X-2 & 325.654 & 38.091 & & & 301.0 & 3.9717 & 146.J & D & 1433.3 & 0.9 & 326.45 & LMXB \\
\hline CYG X-2 & 325.654 & 38.091 & & & 301.0 & 3.9735 & 864.0 & D & 2110.6 & 0.9 & 964.06 & LMXB \\
\hline CYG X-1 & 299.120 & 35.065 & -0.015 & -0.005 & 319.0 & 3.9837 & 1255.4 & B & 1073.3 & 0.8 & 212.04 & MXRB \\
\hline NGC3227 & 155.195 & 20.119 & & & 354.0 & 4.0044 & 1838.2 & $\mathbf{N}$ & 1447.3 & -1.0 & 1.03 & Seyfert 1 \\
\hline SCO430 & 67.675 & -61.546 & & & 362.0 & 4.0501 & 1261.5 & MN & 1049.0 & -1.0 & 1.12 & Cluster \\
\hline CRAB & 82.870 & 21.978 & & & 0.0 & 4.5160 & 1053.3 & $\mathbf{D}$ & 898.6 & -1.0 & 322.38 & SNR \\
\hline CYG X-2 & 325.654 & 38.091 & 0.070 & -0.015 & 208.4 & 4.5437 & 659.2 & D & 1041.0 & 3.4 & 699.02 & LMXB \\
\hline CYG X-2 & 325.654 & 38.091 & & & 209.0 & 4.5516 & 814.6 & D & 1116.4 & 2.5 & 325.55 & LMXB \\
\hline NGC4051 & 180.152 & 44.810 & & & 72.0 & 4.5681 & 2290.7 & $\mathrm{MN}$ & 1027.3 & 2.2 & 1.42 & Seyfert 1 \\
\hline LMC X-1 & 85.023 & -69.768 & & & 327.0 & 4.6117 & 1235.6 & $\mathrm{D}$ & 992.9 & -1.0 & 45.56 & MXRB \\
\hline NGC1566 & 64.722 & -55.056 & & & 308.0 & 4.6262 & 1252.8 & $\mathrm{~N}$ & 1083.5 & -1.0 & 1.09 & Seyfert 1 \\
\hline SN1987A & 83.958 & -69.300 & & & 327.0 & 4.6530 & 244.7 & D & 880.5 & -1.0 & 1.94 & SNR \\
\hline SN1987A & 83.958 & -69.300 & & & 327.0 & 4.6670 & 1365.1 & D & 1111.5 & -1.0 & 4.28 & SNR \\
\hline
\end{tabular}


TABLE 1 -Continued

\begin{tabular}{|c|c|c|c|c|c|c|c|c|c|c|c|c|}
\hline Source & R.A." & Decl. ${ }^{2}$ & R.A. offset ${ }^{\text {b }}$ & Decl. offset ${ }^{b}$ & Roll $^{c}$ & Start $^{d}$ & $\operatorname{Exp}^{e}$ & $D / N^{f}$ & $\mathrm{GR}^{\mathbf{g}} \quad$ & Angle & Rate $^{\mathbf{i}}$ & Class $^{j}$ \\
\hline Q1821 & 275.433 & 64.326 & 2.616 & 0.426 & 181.9 & 4.6998 & 2170.8 & B & 1080.0 & -1.0 & 16.01 & RQ QSO \\
\hline NGC1399 & 54.142 & -35.612 & -0.001 & -0.009 & 124.5 & 5.0236 & 737.0 & B & 1424.6 & 0.5 & 3.33 & Ellip. Galaxy \\
\hline NGC1399 & 54.142 & -35.612 & -0.004 & 0.004 & 122.6 & 5.0495 & 4202.5 & B & 1234.9 & 0.3 & 12.36 & Ellip. Galaxy \\
\hline BY-DRA & 278.190 & 51.680 & -0.057 & 0.090 & 180.2 & 5.1181 & 1126.0 & D & 1109.4 & 5.6 & 3.12 & K Star \\
\hline A2256 & 256.685 & 78.713 & -0.513 & 0.005 & 198.4 & 5.1323 & 2566.1 & MN & 1100.6 & 6.0 & 3.83 & Cluster \\
\hline Q1821 & 275.433 & 64.326 & -0.155 & 0.080 & 178.4 & 5.1620 & 4822.1 & D & 2759.1 & 6.3 & 8.57 & RQ QSO \\
\hline $0614+09$ & 93.595 & 9.156 & & & 60.0 & 5.2252 & 328.3 & D & 1070.1 & -1.0 & 8.32 & LMXB \\
\hline IC443 & 93.583 & 22.817 & 0.200 & -0.077 & 62.5 & 5.3403 & 471.4 & $\mathrm{~N}$ & 1033.1 & 12.0 & 3.73 & SNR \\
\hline IC443 & 93.583 & 22.817 & 0.164 & 0.038 & 62.7 & 5.3458 & 1198.9 & B & 1019.4 & 9.3 & 15.03 & SNR \\
\hline A2256 & 256.685 & 78.713 & & & 280.0 & 5.3801 & 207.4 & D & 998.0 & -1.0 & 5.18 & Cluster \\
\hline PERSEUS & 49.123 & 41.331 & & & 165.0 & 5.3870 & 2166.3 & $\mathrm{MN}$ & 952.8 & -1.0 & 4.93 & Cluster \\
\hline LMC X-1 & 85.023 & -69.768 & & & 40.0 & 5.4642 & 465.2 & $\mathrm{~N}$ & 885.9 & -1.0 & 46.42 & MXRB \\
\hline SN1987A & 83.958 & -69.300 & -0.157 & -0.023 & 38.9 & 5.4697 & 2163.9 & B & 989.4 & 3.6 & 2.12 & SNR \\
\hline LMC X-1 & 85.023 & -69.768 & & & 40.0 & 5.4950 & 267.8 & D & 1043.1 & -1.0 & 44.55 & MXRB \\
\hline A262 & 27.475 & 35.917 & 0.235 & 0.107 & 193.8 & 5.5080 & 2581.3 & B & 996.1 & 13.1 & 1.57 & Cluster \\
\hline EV-LAC & 341.162 & 44.075 & 0.096 & 0.068 & 223.0 & 5.5681 & 2210.5 & B & 1043.2 & 5.8 & 1.58 & M4 Star \\
\hline NGC4151 & 182.004 & 39.684 & & & 81.0 & 5.6160 & 691.2 & D & 1488.3 & 6.0 & 6.31 & Seyfert 1.5 \\
\hline COMA-CL & 194.292 & 28.224 & & & 71.0 & 5.6251 & 250.5 & D & 1114.4 & -1.0 & 8.64 & Cluster \\
\hline $0851+202$ & 132.989 & 20.300 & 0.198 & -0.032 & 291.0 & 5.6593 & 1878.7 & B & 1214.5 & 11.3 & 1.17 & BL Lac \\
\hline NGC2992 & 145.821 & -13.903 & & & 316.0 & 5.6829 & 86.1 & D & 1671.4 & -1.0 & 1.61 & Seyfert 2 \\
\hline A496 & 67.835 & -12.637 & -0.017 & 0.724 & 250.7 & 5.7254 & 760.1 & $\mathrm{~N}$ & 989.4 & -1.0 & 5.85 & Cluster \\
\hline $0114+65$ & 18.674 & 65.026 & 0.018 & 0.018 & 306.8 & 5.7610 & 517.2 & D & 1031.3 & 1.2 & 6.80 & MXRB \\
\hline Н $0538+61$ & 84.566 & 60.834 & -0.005 & -0.005 & 248.1 & 5.7677 & 2458.7 & MN & 1334.6 & 0.3 & 3.74 & CV (AM Her) \\
\hline $0548-32$ & 87.208 & -32.282 & & & 240.0 & 5.8008 & 184.5 & D & 1436.0 & -1.0 & 7.11 & BL Lac \\
\hline N132D & 81.376 & -69.684 & & & 237.0 & 5.8042 & 214.7 & D & 1930.6 & -1.0 & 10.67 & SNR \\
\hline SN1987A & 83.958 & -69.300 & 0.127 & -0.010 & 239.6 & 5.8070 & 1036.8 & D & 1757.4 & 2.8 & 3.16 & SNR \\
\hline N132D & 81.376 & -69.684 & & & 238.0 & 5.8190 & 276.5 & D & 895.4 & -1.0 & 15.95 & SNR \\
\hline MKN3 & 92.452 & 71.053 & -0.059 & -0.010 & 239.2 & 5.8330 & 2310.8 & $\mathrm{~N}$ & 1462.8 & 1.3 & 1.10 & Seyfert 2 \\
\hline NGC253 & 11.274 & -25.561 & -0.004 & -0.026 & 280.0 & 5.8788 & 1671.2 & D & 1073.2 & 1.6 & 2.09 & Starburst \\
\hline NGC4151 & 182.004 & 39.684 & & & 160.0 & 5.9064 & 570.2 & $\mathbf{N}$ & 1779.9 & 4.0 & 8.00 & Seyfert 1.5 \\
\hline NGC4151 & 182.004 & 39.684 & & & 159.0 & 5.9202 & 581.2 & MD & 1105.1 & 4.0 & 6.73 & Seyfert 1.5 \\
\hline NGC4151 & 182.004 & 39.684 & & & 158.0 & 5.9270 & 1181.0 & D & 1871.7 & 0.0 & 8.19 & Seyfert 1.5 \\
\hline RS-CVN & 197.074 & 36.200 & 0.038 & 0.067 & 148.7 & 5.9410 & 741.0 & D & 1445.3 & 4.4 & 20.60 & RSCVn \\
\hline ZET-PUP & 120.457 & -39.861 & 0.108 & 0.018 & 219.0 & 5.9695 & 665.3 & $\mathrm{~N}$ & 1981.9 & 5.1 & 2.63 & OB Sgiant \\
\hline VELA-X & 128.413 & -45.003 & 0.039 & -0.036 & 226.3 & 5.9772 & 1148.9 & B & 1190.1 & 2.7 & 8.61 & SNR \\
\hline GAM-CAS & 13.413 & 60.445 & & & 45.0 & 6.0364 & 60.5 & $\mathrm{~N}$ & 1781.0 & -1.0 & 2.99 & MXRB \\
\hline IC443 & 93.583 & 22.817 & 0.032 & -0.001 & 34.8 & 6.1632 & 1439.7 & MN & 1159.2 & 1.8 & 12.03 & SNR \\
\hline ZET-ORI & 84.559 & -1.968 & 0.071 & 0.004 & 128.8 & 6.2255 & 1028.2 & $\mathbf{N}$ & 1099.2 & 4.3 & 1.57 & O Star \\
\hline ZET-ORI & 84.559 & -1.968 & -0.029 & 0.004 & 129.4 & 6.2374 & 846.7 & B & 1033.0 & 1.7 & 2.83 & O Star \\
\hline CYG X-1 & 299.120 & 35.065 & & & 270.0 & 6.2548 & 805.3 & D & 5559.5 & -1.0 & 144.21 & MXRB \\
\hline CAS-A & 350.292 & 58.540 & & & 195.0 & 6.2665 & 123.6 & D & 1054.6 & -1.0 & 144.46 & SNR \\
\hline PERSEUS & 49.123 & 41.331 & 0.023 & -0.032 & 120.7 & 6.2732 & 2524.5 & $\mathbf{N}$ & 973.9 & 2.2 & 43.62 & Cluster \\
\hline A262 & 27.475 & 35.917 & 0.031 & -0.045 & 135.3 & 6.3378 & 1080.1 & MN & 976.8 & 3.1 & 3.40 & Cluster \\
\hline ALGOL & 46.227 & 40.765 & 0.022 & -0.029 & 123.2 & 6.3514 & 1523.9 & $\mathbf{N}$ & 950.1 & 2.0 & 7.29 & RSCVn \\
\hline $0620-003$ & 95.046 & -0.320 & & & 41.0 & 6.3720 & 172.8 & D & 1054.0 & -1.0 & 1.67 & LMXB \\
\hline VELA X-1 & 135.055 & -40.357 & -0.012 & 0.053 & 32.6 & 6.4132 & 3066.5 & B & 983.4 & 3.2 & 40.81 & MXRB \\
\hline $0212+735$ & 33.208 & 73.595 & -0.314 & -0.068 & 180.9 & 6.4594 & 311.0 & D & 954.0 & 6.7 & 2.73 & RL QSO \\
\hline MKN3 & 92.452 & 71.053 & -0.259 & 0.038 & 125.0 & 6.4650 & 2511.1 & MN & 919.7 & 5.5 & 1.00 & Seyfert 2 \\
\hline
\end{tabular}


TABLE $1-$ Continued

\begin{tabular}{|c|c|c|c|c|c|c|c|c|c|c|c|c|}
\hline Source & R.A. ${ }^{\mathrm{a}}$ & Decl. $^{a}$ & R.A. offset ${ }^{\text {b }}$ & Decl. offset ${ }^{b}$ & Roll $^{c}$ & Start $^{d}$ & $\operatorname{Exp}^{e}$ & $\mathrm{D} / \mathrm{N}^{\mathrm{f}}$ & $G^{8}$ & Angle $^{h}$ & Rate $^{i}$ & Class $^{j}$ \\
\hline 2155-304 & 328.993 & -30.465 & & & 253.0 & 6.5090 & 1123.2 & D & 1061.3 & -1.0 & 16.88 & BL Lac \\
\hline MKN279 & 207.973 & 69.554 & 0.416 & 0.058 & 47.3 & 6.5321 & 475.2 & $\mathrm{~N}$ & 929.0 & 9.4 & 1.25 & Seyfert 1 \\
\hline NGC4051 & 180.152 & 44.810 & & & 67.0 & 6.5562 & 1399.7 & D & 1212.0 & 2.5 & 1.76 & Seyfert 1 \\
\hline M15 & 321.888 & 11.947 & & & 288.0 & 6.5783 & 1078.2 & D & 1064.5 & -1.0 & 17.17 & LMXB \\
\hline NGC4151 & 182.004 & 39.684 & & & 99.0 & 6.6052 & 742.5 & $\mathbf{N}$ & 886.9 & 4.5 & 4.34 & Seyfert 1.5 \\
\hline NGC4151 & 182.004 & 39.684 & & & 98.0 & 6.6210 & 1382.4 & D & 1390.2 & 1.5 & 5.64 & Seyfert 1.5 \\
\hline 2155-304 & 328.993 & -30.465 & 0.022 & -0.050 & 343.1 & 6.6495 & 1503.4 & B & 1282.0 & 3.2 & 37.22 & BL Lac \\
\hline $0836-42$ & 128.912 & -42.714 & 0.043 & 0.032 & 339.2 & 6.6858 & 1611.4 & D & 1508.8 & 2.7 & 20.75 & LMXB \\
\hline NGC1068 & 40.030 & -0.225 & 0.021 & 0.000 & 300.3 & 6.7140 & 2975.4 & $\mathrm{MN}$ & 1264.4 & 1.3 & 2.30 & Seyfert 2 \\
\hline $0548-32$ & 87.208 & -32.282 & 0.088 & 0.022 & 277.7 & 6.7883 & 578.9 & $\mathrm{~N}$ & 2052.6 & 4.7 & 6.07 & BL Lac \\
\hline $0548-32$ & 87.208 & -32.282 & -0.009 & 0.036 & 277.3 & 6.7950 & 804.8 & $\mathrm{~N}$ & 1182.9 & 2.2 & 4.18 & BL Lac \\
\hline $0548-32$ & 87.208 & -32.282 & 0.090 & 0.010 & 276.5 & 6.8044 & 656.4 & $\mathrm{~N}$ & 1040.0 & 4.6 & 5.63 & BL Lac \\
\hline 44IBOOA & 225.535 & 47.848 & 0.081 & 0.001 & 134.9 & 6.8240 & 1672.1 & D & 1434.1 & 3.3 & $4.2 \mathrm{~b}$ & Contact binary \\
\hline X-PER & 58.063 & 30.900 & 0.028 & -0.010 & 332.3 & 6.9917 & 858.3 & $\mathbf{N}$ & 1225.6 & 1.6 & 12.46 & MXRB \\
\hline $1426+428$ & 216.650 & 42.896 & & & 305.0 & 7.0084 & 447.5 & D & 1810.3 & -1.0 & 7.05 & BL Lac \\
\hline $1426+428$ & 216.650 & 42.896 & & & 305.0 & 7.0136 & 371.0 & D & 1962.1 & -1.0 & 6.93 & BL Lac \\
\hline $1426+428$ & 216.650 & 42.896 & & & 305.0 & 7.0183 & 1370.2 & D & 1220.4 & -1.0 & 9.82 & BL Lac \\
\hline $\mathrm{A} 665$ & 126.575 & 66.011 & 0.011 & 0.010 & 299.3 & 7.0398 & 2715.7 & B & 1727.1 & 0.7 & 2.18 & Cluster \\
\hline NGC2110 & 87.443 & -7.467 & 0.029 & 0.005 & 351.6 & 7.1021 & 2488.5 & MN & 1116.0 & 1.8 & 2.10 & Seyfert \\
\hline $0212+735$ & 33.208 & 73.595 & 0.098 & -0.098 & 65.0 & 7.1632 & 673.9 & D & 843.6 & 6.1 & 0.94 & RL QSO \\
\hline $0212+735$ & 33.208 & 73.595 & -0.034 & -0.006 & 65.6 & 7.1778 & 1313.2 & $\mathrm{MN}$ & 1074.4 & 0.7 & 0.95 & RL QSO \\
\hline $1634+706$ & 248.715 & 70.627 & 0.015 & -0.008 & 227.8 & 7.2127 & 3187.6 & B & 998.7 & 0.6 & 1.93 & RQ QSO \\
\hline NGC3227 & 155.195 & 20.119 & & & 350.0 & 7.2602 & 86.4 & D & 1044.9 & -1.0 & 3.83 & Seyfert 1 \\
\hline CEN X-3 & 169.758 & -60.349 & & & 40.0 & 7.2646 & 1640.7 & D & 7209.9 & -1.0 & 105.15 & MXRB \\
\hline Z-CAM & 124.915 & 73.273 & & & 28.0 & 7.2910 & 110.1 & D & 819.4 & -1.0 & 2.55 & CV \\
\hline M82 & 147.935 & 69.907 & 0.136 & -0.023 & 26.9 & 7.2931 & 743.9 & $\mathrm{~N}$ & 898.7 & 3.1 & 2.18 & Galaxy \\
\hline Z-CAM & 124.915 & 73.273 & 0.191 & -0.021 & 49.2 & 7.3025 & 791.4 & $\mathbf{N}$ & 914.1 & 3.5 & 0.74 & CV \\
\hline M82 & 147.935 & 69.907 & 0.143 & -0.025 & 28.2 & 7.3123 & 1123.7 & $\mathbf{N}$ & 978.2 & 3.3 & 2.62 & Galaxy \\
\hline A262 & 27.475 & 35.917 & 0.203 & 0.022 & 149.1 & 7.3514 & 1898.0 & B & 936.0 & 10.0 & 1.99 & Cluster \\
\hline A262 & 27.475 & 35.917 & 0.023 & -0.072 & 149.5 & 7.3753 & 855.4 & MN & 913.8 & 4.5 & 2.62 & Cluster \\
\hline BD61DEG & 163.150 & 60.736 & & & 40.0 & 7.3876 & 121.8 & D & 1085.7 & -1.0 & 1.62 & flare star \\
\hline M49 & 186.808 & 8.276 & 0.176 & -0.003 & 12.8 & 7.3910 & 757.2 & D & 1155.4 & 10.4 & 1.43 & Ellip. Galaxy \\
\hline $3 \mathrm{C} 273$ & 186.639 & 2.329 & 0.206 & 0.010 & 83.9 & 7.4380 & 988.1 & MN & 838.5 & 12.4 & 5.20 & RL QSO \\
\hline $3 \mathrm{C} 273$ & 186.639 & 2.329 & & & 81.0 & 7.4500 & 1537.3 & D & 1080.2 & -1.0 & 8.33 & RL QSO \\
\hline II-PEG & 358.121 & 28.355 & 0.254 & -0.005 & 175.5 & 7.4742 & 841.6 & D & 952.8 & 13.5 & 1.16 & K2 Sgiant \\
\hline II-PEG & 358.121 & 28.355 & 0.008 & 0.016 & 174.4 & 7.4841 & 1675.5 & $\mathrm{~N}$ & 997.7 & 0.5 & 5.49 & K2 Sgiant \\
\hline M82 & 147.935 & 69.907 & 0.170 & -0.011 & 62.9 & 7.5086 & 138.2 & $\mathbf{N}$ & 878.5 & 3.6 & 2.36 & Galaxy \\
\hline $1218+30$ & 184.713 & 30.454 & 0.083 & -0.008 & 41.1 & 7.5150 & 1346.8 & D & 1261.7 & 4.3 & 2.52 & BL Lac \\
\hline A262 & 27.475 & 35.917 & 0.080 & 0.039 & 169.9 & 7.5574 & 1136.3 & $\mathbf{N}$ & 908.5 & 4.5 & 3.04 & Cluster \\
\hline M15 & 321.888 & 11.947 & & & 289.0 & 7.6164 & 345.6 & $N$ & 1178.4 & -1.0 & 2.82 & LMXB \\
\hline M15 & 321.888 & 11.947 & & & 289.0 & 7.6204 & 211.7 & $\mathbf{N}$ & 1080.1 & -1.0 & 20.83 & LMXB \\
\hline TYCHO & 5.6380 & 63.860 & -0.035 & -0.030 & 290.1 & 7.6864 & 895.9 & $\mathbf{N}$ & 952.9 & 2.0 & 47.95 & SNR \\
\hline MKN335 & 0.9384 & 19.925 & 0.044 & 0.016 & 153.7 & 7.7498 & 786.2 & $\mathbf{N}$ & 1020.1 & 2.6 & 1.62 & Seyfert 1 \\
\hline ZET-PUP & 120.457 & -39.861 & -0.063 & 0.158 & 291.3 & 7.7670 & 1095.8 & D & 1855.7 & 9.9 & 1.97 & OB Sgiant \\
\hline $0851+202$ & 132.989 & 20.300 & -0.005 & -0.040 & 320.3 & 7.8160 & 1468.8 & B & 1129.2 & 2.4 & 0.99 & BL Lac \\
\hline $0237-230$ & 39.4700 & -23.368 & 0.055 & -0.088 & 155.8 & 7.8560 & 1408.3 & B & 1189.7 & 6.1 & 1.80 & RL QSO \\
\hline $0237-230$ & 39.4700 & -23.368 & -0.011 & 0.013 & 157.1 & 7.8745 & 1658.4 & B & 1323.7 & 1.0 & 1.19 & RL QSO \\
\hline CAR+WR25 & 5160.671 & -59.413 & 0.169 & 0.032 & 287.4 & 7.9000 & 1433.1 & D & 1687.5 & 5.5 & 8.68 & W-Rayet \\
\hline
\end{tabular}


TABLE 1-Continued

\begin{tabular}{|c|c|c|c|c|c|c|c|c|c|c|c|c|}
\hline Source & R.A. ${ }^{a}$ & Decl. ${ }^{\mathrm{a}}$ & R.A. offset ${ }^{b}$ & Decl. offset ${ }^{b}$ & Roll $^{c}$ & Start $^{d}$ & $\operatorname{Exp}^{e}$ & $\mathrm{D} / \mathrm{N}^{\mathrm{I}}$ & $\mathrm{GR}^{\mathrm{B}}$ & Angle $^{h}$ & Rate $^{i}$ & Class' \\
\hline YY-GEM & 112.858 & 31.980 & 0.062 & -0.012 & 262.1 & 7.9396 & 462.8 & $\mathbf{N}$ & 1845.6 & 3.2 & 2.02 & M binary \\
\hline SIG-GEM & 115.047 & 29.006 & 0.043 & -0.015 & 260.5 & 7.9451 & 1045.5 & $\mathrm{~N}$ & 1168.5 & 2.4 & 4.62 & K1 Sgiant \\
\hline $0212+735$ & 33.208 & 73.595 & 0.040 & 0.117 & 348.0 & 7.9939 & 1856.9 & $\mathrm{~N}$ & 1358.0 & 7.1 & 1.04 & RL QSO \\
\hline 24-UMA & 142.524 & 70.053 & 0.253 & -0.065 & 243.2 & 8.0155 & 723.3 & B & 2022.9 & 6.5 & 3.11 & G4 Sgiant \\
\hline M82 & 147.935 & 69.907 & 0.197 & -0.032 & 269.8 & 8.0536 & 2599.7 & B & 1173.8 & 4.5 & 2.40 & Galaxy \\
\hline $1 \mathrm{E} 1048.5$ & 162.140 & 54.343 & & & 257.0 & 8.0890 & 521.0 & D & 6609.4 & -1.0 & 3.74 & CV (AM Her) \\
\hline ZET-ORI & 84.559 & -1.968 & -0.021 & 0.003 & 359.1 & 8.1424 & 699.5 & $\mathrm{MN}$ & 1395.4 & 1.2 & 2.13 & O Star \\
\hline $1700+64$ & 255.169 & 64.274 & -0.099 & 0.014 & 218.2 & 8.1609 & 1901.1 & D & 1113.3 & 2.7 & 2.13 & RL QSO \\
\hline A2256 & 256.685 & 78.713 & -0.134 & 0.003 & 217.9 & 8.1856 & 1701.3 & $\mathrm{~N}$ & 999.2 & 1.6 & 4.70 & Cluster \\
\hline PUPAB & 125.250 & -42.683 & 0.443 & 0.357 & 69.1 & 8.3875 & 2719.3 & B & 1056.2 & 29.0 & $\mathbf{3 7 . 3 7}$ & SNR \\
\hline PUPAB & 125.250 & -42.683 & 0.333 & 0.101 & 69.1 & 8.4514 & 2937.6 & B & 1079.8 & 15.9 & 48.35 & SNR \\
\hline M87 & 187.073 & 12.667 & 0.018 & 0.207 & 359.9 & 8.5100 & 1712.6 & MN & 914.7 & 12.4 & 9.21 & Radio Galaxy \\
\hline
\end{tabular}

${ }^{a}$ Nominal right ascension and declination of source in decimal degrees (for epoch 1950.0).

${ }^{b}$ Offset of the pointing direction of the A-system telescope from the nominal R.A. and Decl. in decimal degrees (epoch 1950.0), given

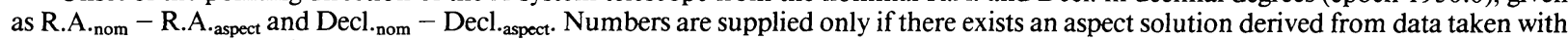
the aspect camera. Specific details about aspect solutions can be obtained through the HEASARC at GSFC.

${ }^{c}$ Roll angle of the A-system telescope $(\phi)$ in degrees. If no aspect solution exists, the roll angle is only an approximate value.

d Start time is given in units of decimal days of MET measured from 1990 December 2, 06:49:01 UT.

${ }^{\mathrm{e}}$ Exposure time in seconds.

${ }^{\mathrm{f}}$ Indicator of shuttle day or night: $\mathrm{N}=$ night; $\mathrm{D}=$ day; $\mathrm{MN}=$ mostly night $(>75 \%)$; $\mathrm{MD}=$ mostly day $(>75 \%) ; \mathrm{B}=$ both, mixed.

${ }^{\mathrm{g}}$ Mean detector guard rate as defined in $\S 5.1$.

${ }^{\mathrm{h}}$ Average off-axis angle $(\Theta)$ in arcminutes as derived from the nominal and aspect R.A.s and Decl.s (cols. [2]-[5]). When an angle but no R.A. or Decl. offset is given, that angle is derived from ray tracing. A value of - 1 indicates that the angle has not yet been calculated. A ray-tracing program is available from the HEASARC that allows observers to determine their own off-axis angles and to confirm our aspect solutions.

${ }^{i}$ Total count rate in all 10 pixels.

j Type of object.

ula data used for calibration are presented in $\S 3.4$, followed by a discussion of the differences between the $\mathrm{A}$ and $\mathrm{B}$ detectors (§3.5). The effective area of the telescope as well as vignetting and flux corrections for off-axis observations are discussed in $\S 3.6$, while the method used to determine the detector efficiencies is presented $\S 3.7$. The current mirror-plus-detector effective area curves for both on- and off-axis observations are discussed in $\S 3.8$. The description of the effective area calibration is followed by a discussion of the flux calibration ( $\$ 4)$. Finally, a complete discussion of contributions to the detector background and background rejection techniques are presented in $\S 5$, along with a prescription for background subtraction $(\S 6)$. The remaining uncertainties in the calibration are summarized in $\S 7$.

\section{THE BBXRT INSTRUMENT AND PERFORMANCE}

An artist's concept of the BBXRT detector and mirror assembly is shown in Figure 1. The instrument was $4.2 \mathrm{~m}$ in length, $1 \mathrm{~m}$ in diameter, and weighed approximately $680 \mathrm{~kg}$. All of the optical devices (i.e., the telescopes, the aspect camera, and the star tracker) were mounted together on the optical plate. During the mission, snapshots of stars were obtained with the aspect camera (a charge injection device with a $4^{\circ} \times$ $6^{\circ}$ field of view) for real-time aspect determination, while the star tracker was used to update the pointing system reference. The X-ray focusing optical system provided a collecting area of $\sim 750 \mathrm{~cm}^{2}$ at $1.5 \mathrm{keV}$ and $\sim 300 \mathrm{~cm}^{2}$ at $7 \mathrm{keV}$ for the entire focal plane (both detectors).

\subsection{The BBXRT Detectors}

The physics of solid-state detectors has been discussed extensively in the literature (e.g., Fraser 1989, and references therein). Each BBXRT detector, designated by "A" or "B," consisted of a silicon block with a grooved surface, segmenting it into 5 spatial elements or "pixels" (Fig. 2, lower panel). The grooves were $0.5 \mathrm{~mm}$ wide and were covered with an opaque mask that also occulted $0.5 \mathrm{~mm}$ of the detector on either side of the groove. The mask covered a nonnegligible area of 1.36 on the sky, but was necessarily placed over the boundaries between the pixels to prevent those events falling near the boundaries from being detected in more than one pixel. Each of the pixels was equipped with its own external amplifier. The radius of the central pixels (designated A0 or B0) was $5 \mathrm{~mm}$ giving a field of view of 3.6, and the total diameter including the outer 4 pixels was $19 \mathrm{~mm}$ or $17: 3$. Since the central detector elements were smaller than the outer elements, they had about onefourth of the background and hence provided greater sensitivity.

The detectors were formed by evaporating $\sim 200 \AA$ of gold as a thin metal p-type contact onto the front, ungrooved, surface of a $\mathrm{Si}(\mathrm{Li})$ n-type semiconductor. A film made of $4000 \AA$ of Parylene $N\left(\mathrm{C}_{8} \mathrm{H}_{8}\right)$ and $2000 \AA$ of aluminum, supported by an $86 \%$ transmitting nickel mesh, was placed in front of each 


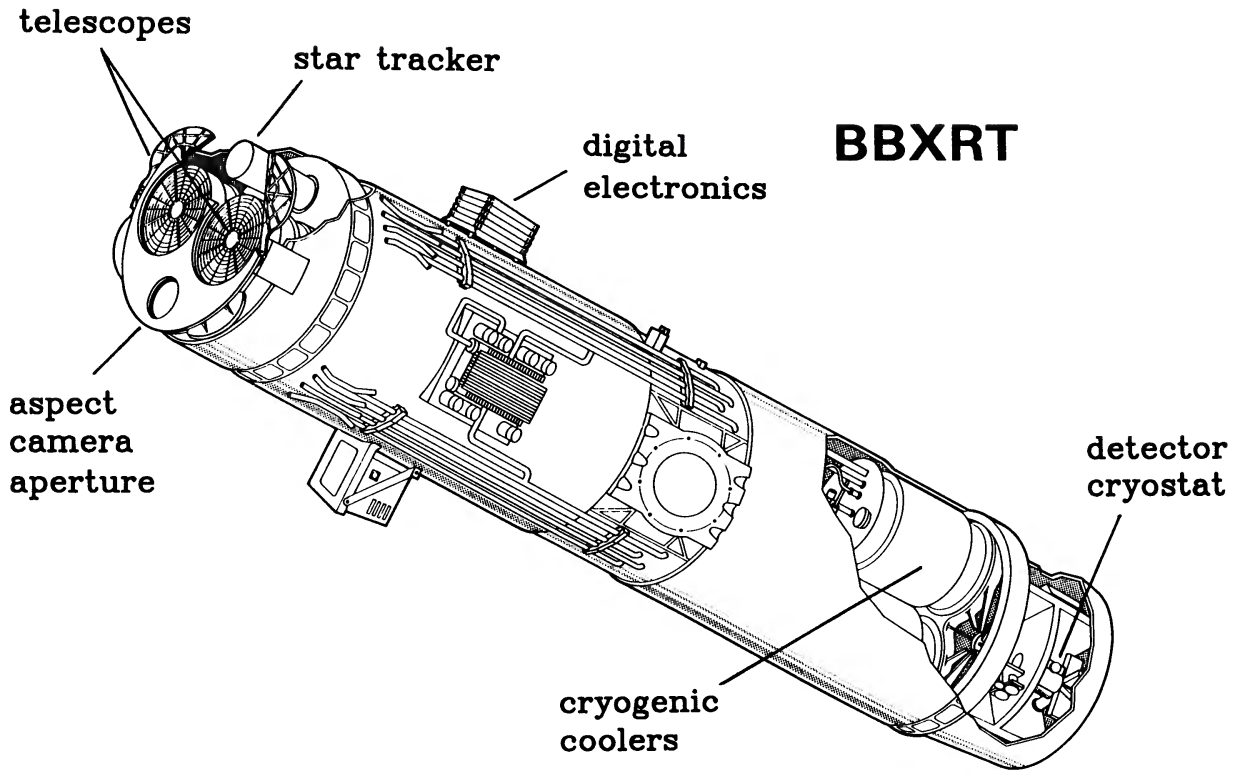

FIG. 1.-Artist's conception of the BBXRT detector and mirror assembly that was flown on the space shuttle Columbia

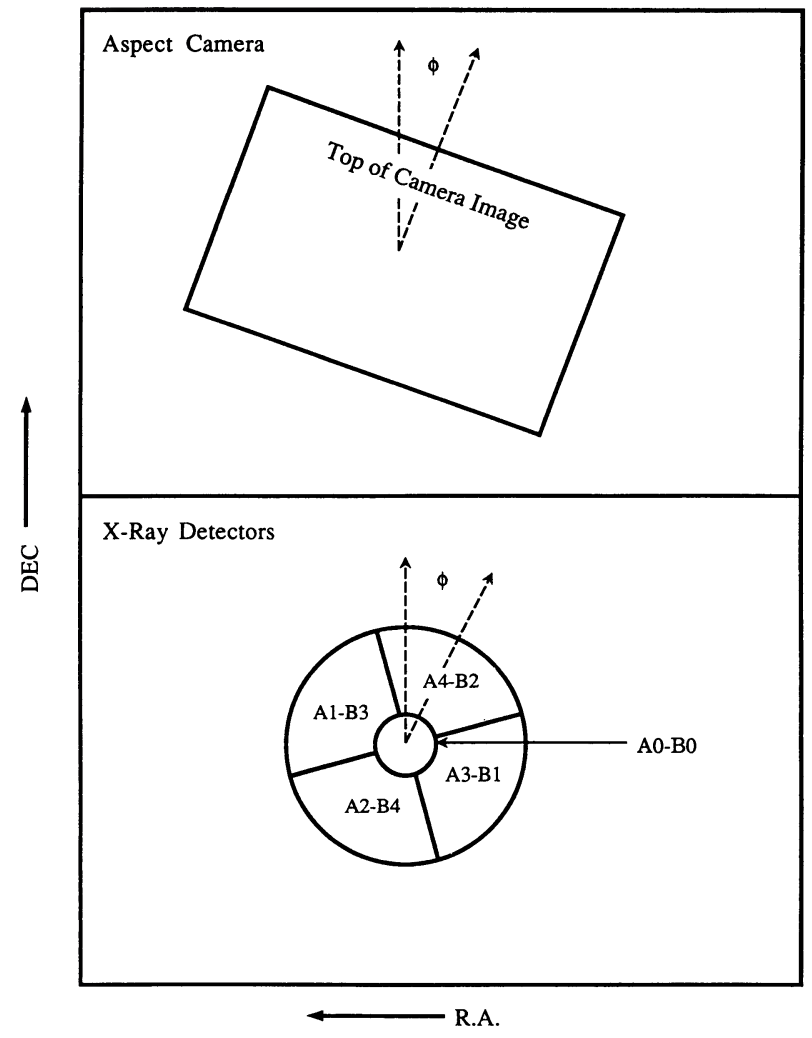

FIG. 2.-Schematic diagram showing the orientation of the BBXRT detectors with respect to the aspect camera. This figure also illustrates the orientation of the pixels on the celestial sphere. The roll angle, $\phi$, is defined such that, when $\phi=0$, the top of the camera and the top of pixel A4 point north. The offset between the A and B detectors (discussed in $\S 2.3$ ) is not shown. detector and served to filter out stray light or any UV component in the focused radiation. The various characteristics of the BBXRT detectors are summarized in Table 2. The detectors resided inside evacuated cryostats that were in thermal contact with cryogenic coolers containing solid argon (Fig. 1) via a 60 $\mathrm{cm}$ long connection. To reduce the number of thermally excited carriers in the silicon and to ensure good operation of the field effect transistor (FET) charge amplifier, the detectors were maintained at a temperature of 108-112 K during operation. A tantalum sheet surrounded the cryostat and was used as a shield against low-energy gamma rays. Outside this tantalum sheet was a plastic scintillator that acted as a charged particle guard and covered over $3 \pi$ sr as viewed from the detector. Also, each detector was equipped with a calibration source holder containing $\mathrm{Fe}^{55}$. During the mission, the calibration source could be moved into the field of view of the detector to monitor changes in the gain or energy resolution.

The central pixels had an on-orbit energy resolution of $\sim 100 \mathrm{eV}$ FWHM $(\Delta E / E \sim 10 \%)$ at $0.9 \mathrm{keV}$ and $\sim 155 \mathrm{eV}$ FWHM at $6 \mathrm{keV}(\Delta E / E \sim 2.5 \%)$, while the outer pixels had a slightly poorer resolution of $\sim 170 \mathrm{eV} \mathrm{FWHM}$ at $6 \mathrm{keV}(\Delta E /$ $E \sim 3.0 \%)$. The signal processing electronics gave each pixel 512 energy channels. The widths of channels $1-255$ are $\sim 16$ $\mathrm{eV}$; the widths of channels $256-511$ are $\sim 32 \mathrm{eV}$; channel 512 is an overscale channel. The physically smaller central detector elements had a lower capacitance, hence a smaller leakage current. This resulted in a low-energy noise threshold of $\lesssim 0.3 \mathrm{keV}$, a few tenths of a keV less than that of the outer elements. The lowest usable channels in A0 and B0 are channel 16 and channel 17 , respectively, which corresponds to $\sim 0.3 \mathrm{keV}$. This ranges from channels 25 to 30 in the outer pixels, which corresponds to $\sim 0.5 \mathrm{keV}$ ( see Table 7 below and $\S 3.8 .2$ ). The discrete low-energy cutoffs are due to setting the discriminator 
TABLE 2

TECHNICAL CHARACTERISTICS OF THE BBXRT DETECTORS

\begin{tabular}{|c|c|}
\hline Characteristic & Value \\
\hline Physical detector thickness ............ & $5 \mathrm{~mm}$ \\
\hline $\mathrm{Si}(\mathrm{Li})$ active area & $17 \mathrm{~mm}$ diameter \\
\hline $\mathrm{Si}(\mathrm{Li})$ depletion depth & $3 \mathrm{~mm}$ \\
\hline $\mathrm{Si}(\mathrm{Li})$ bias voltage $\ldots \ldots \ldots \ldots \ldots \ldots \ldots \ldots$ & $550-600 \mathrm{~V}$ \\
\hline $\mathrm{Si}(\mathrm{Li})$ operating temperature ......... & $108-112 \mathrm{~K}$ \\
\hline 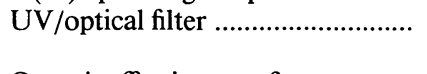 & $\begin{array}{c}\mathrm{C}_{8} \mathrm{H}_{8} \text { and } \mathrm{Al} \text { on an } 86 \% \\
\text { transparent Ni mesh }\end{array}$ \\
\hline $\begin{array}{l}\text { On-axis effective area for one } \\
\text { central pixel }\end{array}$ & $\sim 100 \mathrm{~cm}^{2}$ at $6 \mathrm{keV}$ \\
\hline 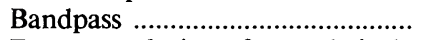 & $0.3-12 \mathrm{keV}$ \\
\hline $\begin{array}{l}\text { Energy resolution of central pixels } \\
\text { (FWHM) }\end{array}$ & $\begin{array}{l}\sim 155 \mathrm{eV} \text { at } 6 \mathrm{keV} \\
\sim 100 \mathrm{eV} \text { at } 0.9 \mathrm{keV}\end{array}$ \\
\hline $\begin{array}{l}\text { Energy resolution of outer pixels } \\
\text { (FWHM) }\end{array}$ & $\begin{array}{l}\sim 170 \mathrm{eV} \text { at } 6 \mathrm{keV} \\
\sim 120 \mathrm{eV} \text { at } 0.9 \mathrm{keV}\end{array}$ \\
\hline Field of view of central pixels ........ & 3.6 diameter \\
\hline 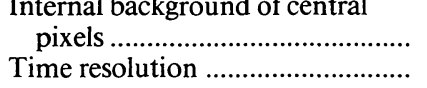 & $\begin{array}{l}1.5 \times 10^{-3} \text { counts } \mathrm{s}^{-1} \mathrm{keV}^{-1} \\
62.5 \mu \mathrm{s}\end{array}$ \\
\hline
\end{tabular}

thresholds just above the energy where the amplifier noise begins to dominate.

\subsection{The X-Ray Mirrors and Point-Response Function}

BBXRT used $10 \mathrm{~cm}$ long conical reflectors that approximated a Wolter type I mirror optical system. There were two adjacent, coaxial, and confocal arrays of cones (the primary and secondary) in each mirror assembly. The BBXRT mirrors were constructed from $0.013 \mathrm{~cm}$ thick aluminum foil that was bent into truncated cones and then dipped in acrylic resin. The function of the resin was to smooth the foil on small scales. The resultant rms microroughness was less than $3 \AA$. The reflecting surface was created by vacuum-depositing approximately 500 $\AA$ of gold on the resin (Serlemitsos 1988). Each mirror assembly consisted of 118 reflectors, and the focal length of the mirror system was $3.77 \mathrm{~m}$. The mirror performance is summarized by Petre et al. (1992), and the mirror fabrication and mirror parameters are described in detail by Serlemitsos (1988) and Serlemitsos et al. (1991).

Although the nested foils gave a large throughput over a large bandpass, the telescope provided only a modest spatial resolution of 1.5 half-power radius (HPR). While this is partially due to the conical approximation to a Wolter 1 geometry, the 12 " HPR intrinsic resolution of the design was far better than that obtained. The prime contributor to the degraded resolution was millimeter-scale ripples on the foil surface that resulted from the manufacturing process and were inadequately covered by the thin lacquer coating. A secondary source of blurring was the inaccurate mechanical fixturing of the foils.

We have determined the beam shape for the mirrors by comparing the preflight point-spread function (PSF) with flight data. This was done by calculating the fraction of source counts expected in each pixel assuming a certain beam shape. For bright sources that are closely aligned with the optical axis, the in-flight data show more power in the outer elements than expected for a single Gaussian profile. At the focal plane, the integral power (beam profile) for each telescope is characterized by

$$
P(<\Theta)=A \operatorname{erf}\left(\Theta / \sigma_{1}\right)+B \operatorname{erf}\left(\Theta / \sigma_{2}\right),
$$

where erf stands for error function, $P(<\theta)$ is the total power that is contained within an angular radius of $\Theta \operatorname{arcmin}, \sigma_{1}=$ 1.8 , and $\sigma_{2}=5.8$. The first term in equation (1) represents the beam core, which contains $65 \%$ of the flux; the second term represents a halo, which contains $35 \%$ of the flux. For a source observed on axis: $\sim 62 \%$ of the counts that reach the detector fall in the central pixel, $\sim 21 \%$ fall on the mask between the pixels, and $\sim 17 \%$ fall into the outer pixels. For a source that is observed near the edge of a pixel or on the mask, a larger fraction of photons is lost to the mask (up to $50 \%$ ). It is necessary to correct for this loss when measuring absolute fluxes (see $\S 3.6$ ). The energy dependence of the PSF is discussed in $\S 3.8 .3$.

\subsection{Mission Description and Instrument Performance}

BBXRT was launched on board the space shuttle Columbia (STS-35) on 1990 December 2, at 01:49:01 eastern standard time (UT 336/06:49:01), which defines a mission elapsed time (MET) of 0.0. The combined shuttle payload also contained the Hopkins Ultraviolet Telescope, Goddard's Ultraviolet Imaging Telescope, and the Wisconsin Ultraviolet Photopolarimeter and is described in detail by Blair \& Gull (1990). The BBXRT instrument was initially turned on about $3 \mathrm{hr}$ after launch (MET $=0.13$, given in decimal days), and the detector system remained active and performed flawlessly until about $6 \mathrm{hr}$ before reentry. During the mission, the instrument alignment remained unchanged and the temperature of the $\mathrm{A}$ and B cryostats remained stable at values of $108 \mathrm{~K}$ and 112 $\mathrm{K}$, respectively. None of the other instrument housekeeping parameters that were recorded during the mission (such as the detector voltages and the cryogen pressure and temperature) varied outside of their nominal values.

The telescope was mounted on the Two-Axis Pointing System (TAPS), which in turn was secured to the bay of the shuttle orbiter. To observe a target, the shuttle had to acquire and to maintain an attitude that allowed the source to stay within the TAPS gimbal range of $\pm 17^{\circ}$ for each axis (Serlemitsos et al. 1991). There were no stable pointings during the first $60 \mathrm{hr}$ of the mission because of an improperly compensated gyro drift rate that allowed TAPS to drift off target. Once this drift was removed TAPS pointed stably, having a drift rate of less than $0 " 1 \mathrm{~s}^{-1}$. However, the absolute accuracy to which TAPS could acquire targets was only $2^{\prime}-5^{\prime}$. This meant that for many observations real-time "tweaks" were needed to center a target. For observations performed during orbit night, attitude solutions were obtained using the aspect camera, with a typical accuracy of $\sim 1^{\prime}$. For observations performed during orbit day, it is possible from TAPS and shuttle position data to determine to within $5^{\prime}$ where BBXRT was pointing. However, by comparing the ratios of count rates in different detector elements to Monte Carlo simulations of the distribution of events in the 
focal plane consistent with the mirror point-response function ( 2.2 ), we are able to obtain fairly accurate off-axis positions for daytime observations. The uncertainty in each estimate is typically 0.5 for sources with medium to high count rates located within $5^{\prime}$ of the center of the field of view, and $\sim 1^{\prime}-2^{\prime}$ for weak sources or sources located more than $5^{\prime}$ from the center. Although some off-axis angles have been calculated for sources lacking an aspect solution, this has not yet been done for $\sim 37 \%$ of the observations listed in Table 1 . The necessary raytracing software is currently available through the High Energy Astrophysics Science Archive Research Center (HEASARC) located at Goddard Space Flight Center.

Figure 2 illustrates the approximate orientation of the BBXRT detectors with respect to the aspect camera and projected onto the celestial sphere. The outer pixels of the A detector are labeled A1 to A4 counterclockwise on the sky. The telescope roll angle, $\phi$ (Table 1), increases counterclockwise and is defined such that, when $\phi=0$, the top of the camera and the top of pixel A4 point north. The B detector is rotated by $180^{\circ}$ with respect to the $A$ detector such that $A 1$ and $B 3$ observe roughly the same area of the sky. Postflight analysis indicates a slight offset between the A and B telescopes (not shown in Fig. 2 ). The mean displacement of B from $A$ is 0.822 at an azimuth of $-63^{\circ} .24$. This azimuth is measured arbitrarily from the B3/ $\mathrm{B} 4$ boundary and represents a $63^{\circ}$ rotation through $\mathrm{B} 4$ toward $\mathrm{B} 1$. In other words, for a source centered in A0, the distribution of counts in the outer B pixels is maximized in B4. In general, this displacement leads to a lower count rate in $B$, since sources centered in A will be offset in B and a larger fraction of counts will fall on the B mask. A correction to account for the difference in observed count rates between $A$ and $B$ is not included in the effective area calculations. Therefore, all fluxes are currently measured with respect to the A system. Since the A system axis is taken as the nominal pointing position of the telescope, the term "on axis" refers to a source that is centered in the A detector.

\section{CALIBRATION}

\subsection{Response Function}

A $\mathrm{Si}(\mathrm{Li})$ detector response function has three basic components. First, the response function contains a Gaussian-shaped photopeak. Second, for incident photons having an energy of $h \nu>1.84 \mathrm{keV}$, a Gaussian-shaped escape peak (due to the escape of Si K X-rays) is observed $1.74 \mathrm{keV}$ below the main photopeak. Finally, there are non-Gaussian components that occur in the response function below the full-energy peak of the main Gaussian. The physics of the non-Gaussian components is not well understood, but they represent charge loss mechanisms in the detector that result in an underestimation of the incident photon energy.

The shape of the response function for a monoenergetic photon can be described by

$$
F(i)=G(i)+\operatorname{Esc}(i)+\mathrm{PC}_{n}(i), \quad n=1,2,3,
$$

where $i$ is the detector channel number, $G(i)$ represents the main Gaussian photopeak, Esc $(i)$ represents the escape peak, and $\mathrm{PC}_{n}(i)$ represents the partial charge collection. The partial charge collection is a fairly complicated function (e.g., Fig. 2 in Krumrey, Tegeler, \& Ulm 1988). There are as many as three major components to the partial charge: a step function at energies lower than the Gaussian photopeak $\left(\mathrm{PC}_{1}\right)$, a low-energy wing on the Gaussian photopeak $\left(\mathrm{PC}_{2}\right)$, and a tail that rises toward very low energies $\left(\mathrm{PC}_{3}\right)$. The specific shapes and magnitudes of these components vary from detector to detector (Shima et al. 1983), and they depend mainly on the thickness of the silicon dead layer. Further discussion of the partial charge collection in the BBXRT detectors is presented in $\S 3.3$.

\subsection{The Detector Gain and Resolution}

The channel-to-energy relation was determined using $\mathrm{Mn}$ $\mathrm{K} \alpha(5.895 \mathrm{keV})$ and $\mathrm{Mn} \mathrm{K} \beta(6.490 \mathrm{keV})$ from the in-flight $\mathrm{Fe}^{55}$ calibration source along with the lower energy atmospheric fluorescence lines of $\mathrm{Ar}(2.956 \mathrm{keV})$ and $\mathrm{O}(0.525$ $\mathrm{keV})$, as well as Si XIII (1.865 keV) and Mg XI (1.348 keV) from scattered solar $\mathrm{X}$-rays. The gain is described by a quadratic function: $y=A+B x+C x^{2}$. Here, $y$ is the channel in which the line peak is observed when fit with a Gaussian and $x$ is the expected line energy. The gain was determined after channel doubling was properly accounted for. The best-fit gain coefficients for each pixel are listed in Table 3. The three columns labeled Start, Middle, and End give the lower energy boundary of the lowest channel (channel 1), the energy where the channel size doubles (channel 256), and the upper energy boundary of the highest channel (channel 512), respectively. The measured uncertainty in the gain at $5.9 \mathrm{keV}$ is less than 1 channel $(<32 \mathrm{eV}$ or $<0.5 \%)$ for all pixels.

We have assumed atomic oxygen $\mathrm{K} \alpha$ fluorescence to be at an energy of $0.525 \mathrm{keV}$, which is the value given by Bearden (1967). However, there are discrepancies in the literature as to the correct value for the oxygen K-edge energy, which may affect the assumed value of the fluorescence line. Bearden \& Burr (1967) find the threshold energy $\left(E_{\mathrm{K}}\right)$ for photoejection of an electron from the $\mathrm{K}$ shell to be $0.532 \mathrm{keV}$, which places the edge at $\sim 0.537 \mathrm{keV}$. However, Gould \& Jung (1991) calculate $E_{\mathrm{K}}$ for atomic oxygen to be $0.546 \mathrm{keV}$. Scaling up the oxygen fluorescence line by the same amount places it at 0.540 $\mathrm{keV}$. This would yield a remaining systematic offset of $15 \mathrm{eV}$ ( 1 channel width) in the gain at low energies and would primarily impact the analysis of soft X-ray spectra with a high signal-to-

TABLE 3

GAIN PARAMETERS

\begin{tabular}{lcccccc}
\hline \hline Detector & A & B & C & Start $(\mathrm{keV})$ & Middle $(\mathrm{keV})$ & End $(\mathrm{keV})$ \\
\hline A0 & -3.540 & 66.529 & -0.238 & 0.060731 & 3.949430 & 12.131009 \\
A1 & -1.262 & 65.518 & -0.186 & 0.026908 & 3.963552 & 12.169284 \\
A2 & -2.090 & 66.181 & -0.273 & 0.039139 & 3.956777 & 12.264119 \\
A3 & -2.186 & 65.432 & -0.215 & 0.041057 & 3.990546 & 12.273397 \\
A4 & -2.970 & 66.774 & -0.245 & 0.051973 & 3.927410 & 12.089725 \\
B0 & -3.030 & 66.123 & -0.181 & 0.053385 & 3.952595 & 12.034285 \\
B1 & -0.772 & 65.278 & -0.246 & 0.019477 & 3.985723 & 12.360293 \\
B2 & -0.641 & 66.084 & -0.237 & 0.017255 & 3.931424 & 12.170003 \\
B3 & -1.751 & 65.828 & -0.218 & 0.034192 & 3.959848 & 12.193321 \\
B4 & -0.599 & 65.697 & -0.204 & 0.016736 & 3.946548 & 12.166389 \\
\hline
\end{tabular}

NOTE.-The gain equation is $y=A+B x+C x^{2}$, where $x$ is the true line energy and $y$ is the channel in which the line peaks. 
noise ratio, such as provided by observations of the bright Earth $(\S 5.4)$.

The resolution of a $\mathrm{Si}(\mathrm{Li})$ detector can be described by the relation $\sigma=\left[(F / \omega) E+\sigma_{A}^{2}\right]^{1 / 2}$ where $F$ is the fano factor, $\omega$ is the mean energy necessary to create one electron-hole pair, and $\sigma_{A}^{2}$ is the variance due to the noise in the electronics (Fraser 1989). The Gaussian response widths were initially measured using photopeaks for $\mathrm{K} \alpha$ and $\mathrm{K} \beta$ and the escape peak for $\mathrm{K} \alpha$ from preflight $\mathrm{Fe}^{55}$ and $\mathrm{Ca}^{41}$ calibration data (the photopeaks of $\mathrm{K}$ from $\mathrm{Ca}^{41}$ are at 3.31 and $3.59 \mathrm{keV}$ ). The calibration lines were modeled with a Gaussian (using a diagonal response matrix), and the results were fit with the relation $y=(A x+$ $B)^{1 / 2}$, where $y$ is the Gaussian $\sigma$ and $x$ is the line energy in $\mathrm{keV}$. The best-fit coefficients for each pixel are given in Table 4. Figure 3 shows the measured FWHM versus energy for the central A pixel. The FWHM resolution is $\sim 155 \mathrm{eV}$ at $5.9 \mathrm{keV}$ in both central pixels and $\sim 170 \mathrm{eV}$ at $5.9 \mathrm{keV}$ in the outer pixels. The in-flight resolution was determined to be the same as the preflight resolution for $\mathrm{Fe}^{55}$.

\subsection{Partial Charge Collection}

Deviations from Gaussian response shapes in $\mathrm{Si}(\mathrm{Li})$ detectors have been extensively discussed in the literature (Shima et al. 1983; Campbell et al. 1985; Yacout, Gardner, \& Verghese 1986; Inagaki, Shima, \& Maezawa 1987). The intensity of the sum of the non-Gaussian-peak components correlates with the size of the detector dead layer, which is generally $0.1-0.2$ $\mu$ m thick (Shima et al. 1983; Joy 1985). The mean absorption depth of an X-ray, which can be written as $\lambda=1 / \rho$, where $\rho$ is the linear absorption coefficient, ranges from $\sim 0.1 \mu \mathrm{m}$ at 0.4 $\mathrm{keV}$ to $14 \mu \mathrm{m}$ at $1.8 \mathrm{keV}$ (just before the Si edge). This is only slightly larger than the dead layer thickness. Therefore, soft Xrays are typically absorbed close to the front of the detector. Partial charge collection and charge trapping can occur when some of the charge carriers diffuse into the dead layer before being accelerated toward the anode by the applied bias voltage. Partial charge collection can also occur from the escape of Auger electrons or photoelectrons that are emitted near the boundary between the dead layer and the depletion region. The fraction of charge that is lost increases as a function of the ratio of the dead layer thickness to the mean absorption depth $(D /$ ג) (Joy 1985; Shima et al. 1983). The partial charge fraction

TABLE 4

RESOLUTION COEFFICIENTS

\begin{tabular}{lcc}
\hline \hline Detector & $\mathrm{A}$ & $\mathrm{B}$ \\
\hline $\mathrm{A} 0$ & $4.48940 \mathrm{E}-04$ & $1.71804 \mathrm{E}-03$ \\
$\mathrm{~A} 1$ & $4.65352 \mathrm{E}-04$ & $2.13648 \mathrm{E}-03$ \\
$\mathrm{~A} 2$ & $4.55287 \mathrm{E}-04$ & $2.32979 \mathrm{E}-03$ \\
A3 & $4.71554 \mathrm{E}-04$ & $2.08003 \mathrm{E}-03$ \\
A4 & $4.85625 \mathrm{E}-04$ & $2.57638 \mathrm{E}-03$ \\
B0 & $5.61384 \mathrm{E}-04$ & $1.23391 \mathrm{E}-03$ \\
B1 & $5.53610 \mathrm{E}-04$ & $1.82161 \mathrm{E}-03$ \\
B2 & $5.28301 \mathrm{E}-04$ & $2.01821 \mathrm{E}-03$ \\
B3 & $5.19983 \mathrm{E}-04$ & $1.95085 \mathrm{E}-03$ \\
B4 & $4.95977 \mathrm{E}-04$ & $2.12448 \mathrm{E}-03$ \\
\hline
\end{tabular}

NOTE.-The energy resolution $(\sigma$ in $\mathrm{keV})$ is given by $y=(A x+$ $B)^{1 / 2}$, where $y$ is the Gaussian $\sigma$ and $x$ is the line energy in $\mathrm{keV}$.

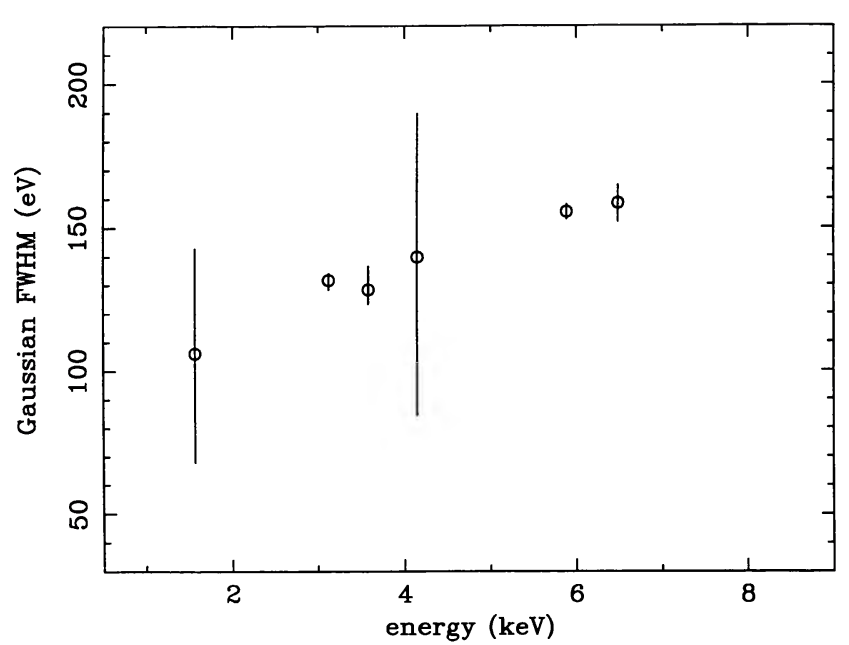

FIG. 3.-Plot of the measured Gaussian FWHM from preflight calibration data vs. energy for the central A detector pixel (A0). The points with the largest error bars were obtained from the $\mathrm{K} \alpha$ escape peaks.

also exhibits a sharp jump at the silicon $\mathrm{K}$ edge where there is a large decrease in the mean absorption depth (Joy 1985; Inagaki et al. 1987).

Utilizing preflight calibration data, we modeled the partial charge in the detector as a step function (convolved with a Gaussian) starting at the energy of the incident X-ray and extending to zero energy. The shape is given by

$$
S(E)=\frac{1}{2} \operatorname{erf}\left(\frac{E-E_{c}}{\sqrt{2 \sigma}}\right)
$$

After the mission, the magnitude of the step was adjusted to approximate the partial charge that was observed for the Crab Nebula as well as the oxygen fluorescence airglow line ( $§ 3.7$ ). Figure 4 illustrates the current model for the response function in pixels $\mathrm{B} 0$ and $\mathrm{B} 3$, compared with potassium $\mathrm{K} \alpha$ and $\mathrm{K} \beta$ from $\mathrm{Ca}^{41}$ obtained from preflight calibration runs. The $\mathrm{A} 0$ data are not shown but are similar to the B0 data. Pixel B3 was chosen to illustrate the partial charge in the outer B pixels, which has a larger tail at less than $0.7 \mathrm{keV}$ than the partial charge in the outer A pixels. Clearly, our step function model does not properly account for the shape of the partial charge distribution. First, a wing on the low-energy side of the main photopeak is visible in Figures $4 a$ and $4 b$. However, since it amounts to less than $5 \%$ of the amplitude of the photopeak and the BBXRT signal-to-noise ratio is not sufficient to observe wings on emission lines from celestial sources, we have not included the wing in the response function. The partial charge distribution also rises toward low energies. The low-energy tail is clearly visible in the B detector when observing bright celestial sources such as the Crab Nebula (see § 3.5). However, we found from comparing the preflight calibration data (e.g., Fig. 4 ) with the Crab data that the relative magnitude of the lowenergy tail as compared with the step function (eq. [3]) appears to be larger in the calibration data. Because of the uncertainties inherent in fitting the Crab to determine the magnitude of this tail for celestial sources, we have presently not included 

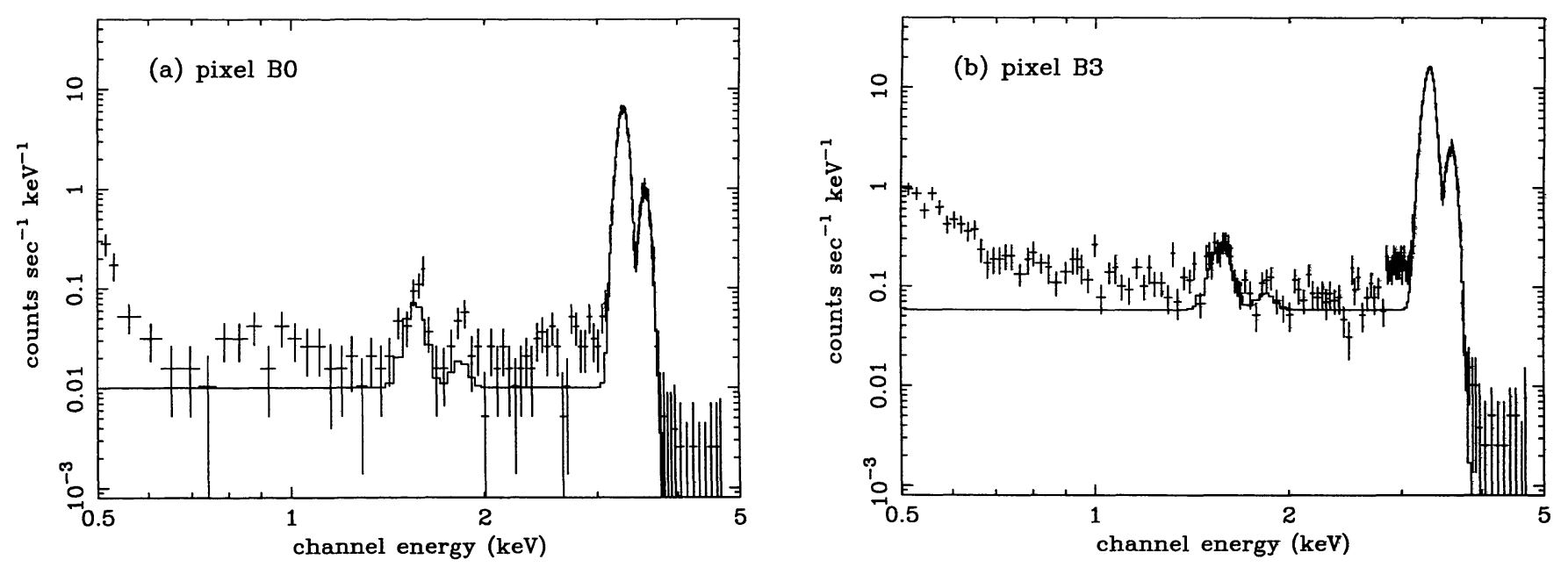

FIG. 4. - Preflight calibration data fit with the present BBXRT response function (solid line): $(a)$ potassium $\mathrm{K} \alpha$ and $\mathrm{K} \beta$ from $\mathrm{Ca}{ }^{4 \mathrm{I}}$ in pixel $\mathrm{B} 0$ and ( $b$ ) potassium $\mathrm{K} \alpha$ and $\mathrm{K} \beta$ from $\mathrm{Ca}^{41}$ in pixel $\mathrm{B} 3$.

it in our response function. However, not including the tail causes difficulty in attempting to adjust the effective area curve at low energies $(\S 3.7)$. The lack of a correct model of the partial charge distribution remains one of the largest uncertainties in the effective area calibration.

\subsection{The Crab Nebula}

Observations of the Crab Nebula (a standard X-ray calibration target) were made four times during the mission, twice approximately on axis and twice well off axis. Light curves of the day 3 observation in the A pixels are shown in Figure 5. The Crab Nebula was first observed for 2300 seconds at $~ 3.0$ off axis, with the source flux highest in pixels A1 and B3. The position of the Crab on the detector during this time is illustrated in Figure 6. An attitude adjustment that began at 3.054 days MET placed the Crab in pixels A0 and B0. This observa-

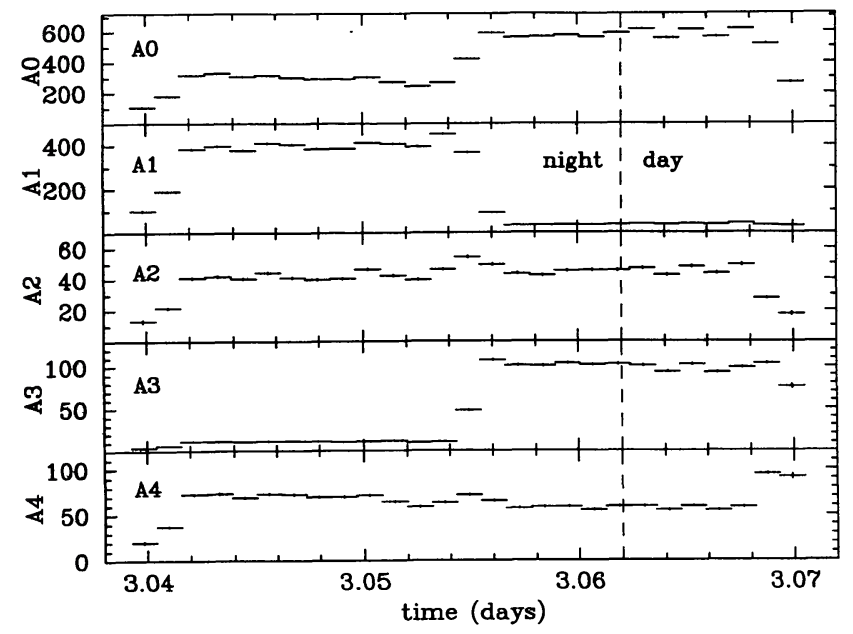

FIG. 5.- - Light curves of the Crab observation from the A detector pixels on day 3. The source pixel changes from pixel A1 to pixel A0 at 3.054 days MET. tion is designated "on axis" for purposes of calibration, although the source was offset by about 1 ' toward pixel A3. The first half of the on-axis observation was performed during orbit night, while the second half was performed during orbit day (Fig. 5).

The Crab Nebula X-ray spectrum can be well described by a single absorbed power law, $N(E) \propto E^{-\mathrm{r}} e^{-\sigma(E) N_{\mathrm{H}}}$, over the 1$10 \mathrm{keV}$ energy band (Seward 1992; Weaver 1993, and references therein). The photon index is well known, with the average value in the literature being $\Gamma=2.1$. There is, however, some disagreement over the observed value of the hydrogen column density $\left(N_{\mathrm{H}}\right)$ along the line of sight to the Crab. The measured values for $N_{\mathrm{H}}$ are not all consistent, and the discrepancy is rather high in a few cases (see Seward 1992; Weaver 1993, Table 3.4; and references therein). The mean value of the column density from the literature is $N_{\mathrm{H}}=2.9 \pm 0.4 \times$ $10^{21} \mathrm{~cm}^{-2}$.

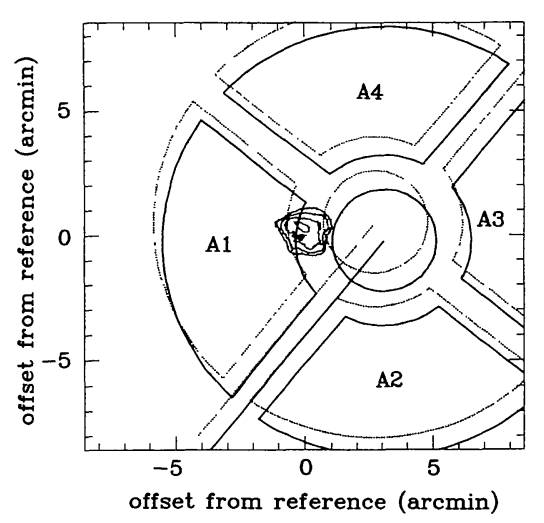

FIG. 6.-BBXRT pixels superposed on an Einstein Observatory IPC image of the Crab Nebula. This represents the orientation of the detectors during the off-axis observation on day 3 (3.04-3.05 days MET). The A pixels are drawn with solid lines, and the B pixels are drawn with dotted lines. This figure illustrates the offset between the A and B detectors that is described in $\S 2.3$. 
Complexities in the Crab's spectral and spatial characteristics have been observed at both high and low energies. The spectrum flattens at high energies due to the contribution from the pulsar. This effect is $\sim 10 \%$ at $10 \mathrm{keV}$ (Toor \& Seward 1977). At energies below $2 \mathrm{keV}$, the angular size of the Crab increases. This has been attributed to interstellar dust that causes low-energy photons to be scattered out of the central regions into a dust halo. The angular diameter of the halo is $8.3 E^{-1}$ arcmin and the fraction of the X-rays scattered into the halo is $0.066 E^{-2}$, where $E$ is measured in $\mathrm{keV}$ (Schattenburg \& Canizares 1986). Eleven percent of the total $0.5-3.5 \mathrm{keV}$ flux measured with the Einstein Observatory IPC appears at a radius of greater than 1'.6 (Mauche \& Gorenstein 1989).

Because the size of the BBXRT central pixel (3.'6 diameter) is comparable to the extent of the Crab at medium energies ( $2^{\prime}$ diameter at $4 \mathrm{keV} ; 16^{\prime}$ diameter at $0.5 \mathrm{keV}$ ), we expect to see a higher fraction of soft photons in the outer pixels than in the central pixels when the Crab is roughly centered in A0. Similarly, we expect to see relatively more soft flux in $\mathrm{A} 0$ when the Crab is 3.0 off axis than when the Crab is roughly centered in A0. Figure 7 shows the ratio of the $\mathrm{A} 0$ spectrum when the $\mathrm{Crab}$ is off axis to the $\mathrm{A} 0$ spectrum when the Crab is on axis, normalized at $\sim 2 \mathrm{keV}$. We do not find the expected increase in the flux ratio at low energies, although a decrease is observed in the ratio of the spectra at high energies due to the off-axis vignetting of the mirrors ( $\S 3.6$ below). A deficiency of counts in the central pixels due to dust scattering is not required in the spectral fits either. When the Schattenburg \& Canizares model of the dust-scattering halo is included in the fit to the on-axis A0 Crab spectrum, the column density decreases from $3 \times 10^{21}$ to $2.7 \times 10^{21} \mathrm{~cm}^{-2}$ (cross sections from Morrison \& McCammon 1983 ) but the fit is not statistically improved. In addition, when an absorbed power-law model is fitted to spectra from the outer A pixels when the Crab is on axis, we find $N_{\mathrm{H}}=3 \times$ $10^{21} \mathrm{~cm}^{-2}$, which is similar to $N_{\mathrm{H}}$ measured in $\mathrm{A} 0$. We conclude that any effects of the dust-scattering halo are less than the uncertainty in the hydrogen column to the Crab, so for simplicity we do not include a dust-scattering halo in our cali-

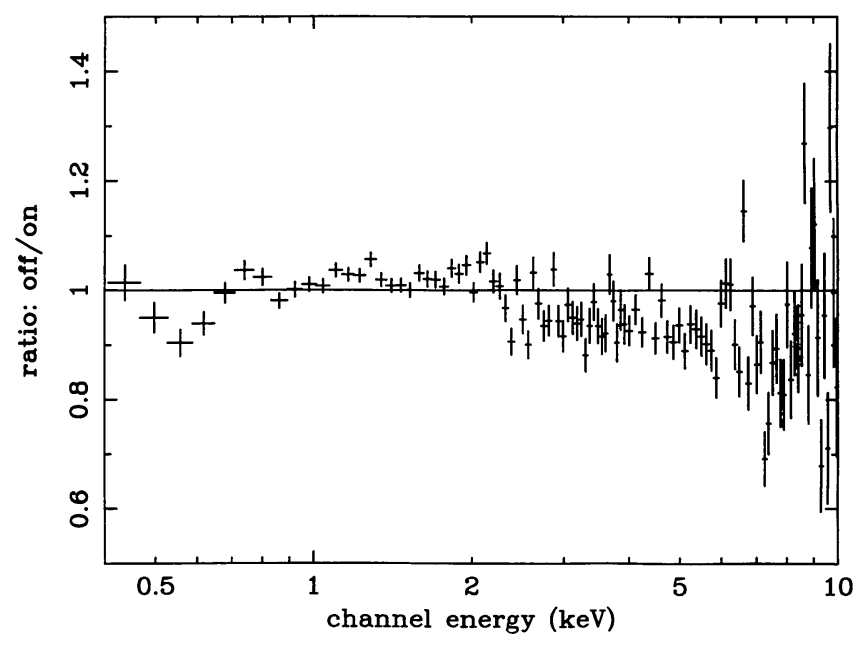

FIG. 7.- Ratio of off-axis to on-axis observations of the Crab Nebula in the central A pixel. The data were normalized at $2 \mathrm{keV}$. The "feature" at $\sim 0.5 \mathrm{keV}$ appears to be variable and is discussed in $\S 3.8 .1$. bration model. The effective areas for the two central pixels are refined based on fitting the Crab spectrum to an absorbed power law with $\Gamma=2.10$ and a neutral hydrogen column density of $3.0 \times 10^{21} \mathrm{~cm}^{-2}$.

\subsection{Differences between Detectors}

Fewer counts are observed in the B detector than in the A detector below about $2-3 \mathrm{keV}$ for all detector elements. Figure 8 illustrates this effect for the Crab in the central pixels. During the mission it was thought that the decreased efficiency in B at low energies was due to the accumulation of ice on the surface of the detector. However, postflight calibrations performed at GSFC and the National Institute for Standards and Technology discredit the hypothesis that the B detector could be covered with ice because the relative deficiency of counts persists even after a deicing of the detectors. Also, the extra absorption is not likely due to excess Parylene in the B filter because the decreased efficiency occurs even at energies of $2 \mathrm{keV}$ (Fig. 8). We therefore conclude that the $\mathrm{B}$ detector must be covered with a thicker gold surface contact layer than the A detector ( $\$ 3.7$ and Table 5).

Another difference between the two detectors is in the magnitude and the shape of the low-energy tail of the partial charge collection in the outer pixels. Figure 9 shows Crab spectra from the A1 and B3 pair of outer pixels when the Crab is on axis. Although between 0.7 and $2.0 \mathrm{keV}$ B has fewer counts than A (due to the excess gold on the B detector), the B3 spectrum rises below $0.7 \mathrm{keV}$ to meet the $\mathrm{A} 1$ spectrum. This is due to a large low-energy tail contribution to the partial charge in the outer B pixels, which is also present in the B detector preflight calibration data (Fig. 4) but not similarly observed in the outer A pixels.

\subsection{The Mirror Areas and Flux Corrections}

The density of the vacuum-deposited gold on the mirror surface is less than that of solid gold. This has the effect of reducing the reflectivity at high energies. However, we had no direct way of measuring the density of the gold on the mirrors. Hence, the effective gold density, $\rho_{\text {eff }}=C \rho_{\text {gold }}$, was treated as a free parameter in the effective area calculations. The on-axis mirror effective area curve was generated by ray tracing (Petre \& Serlemitsos 1988) using a gold density fraction of $C=0.87$, assumed to be the same for both telescopes. The value of $C=$ 0.87 was derived from an early fit to the Crab before absorption due to the Ni mesh in front of the detector was included in the detector efficiency calculations. After a first-order correction for the Ni absorption was included, the original effective area curve gave a slope that was too steep for the on-axis Crab observation in A0. Since the high-energy efficiency of the telescope depends on the gold density, we were able to obtain a more reasonable slope of 2.1 by reducing $C$ from 0.87 to $0.84 .^{5}$ In $\mathrm{A} 0$, the $2-10 \mathrm{keV}$ fit to the Crab after reducing the gold density fraction gave the expected values of $N_{\mathrm{H}}=3 \times 10^{21}$ $\mathrm{cm}^{-2}$ and $\Gamma=2.1$.

\footnotetext{
${ }^{5}$ This flattening was done before a possible explanation was found for a "too steep" Crab slope. A steeper apparent slope in A0 can be caused by energy-dependent scattering of the mirrors $(\S 3.8 .3)$. This implies that the gold density fraction may actually be 0.87 and that we have overcorrected the effective area.
} 


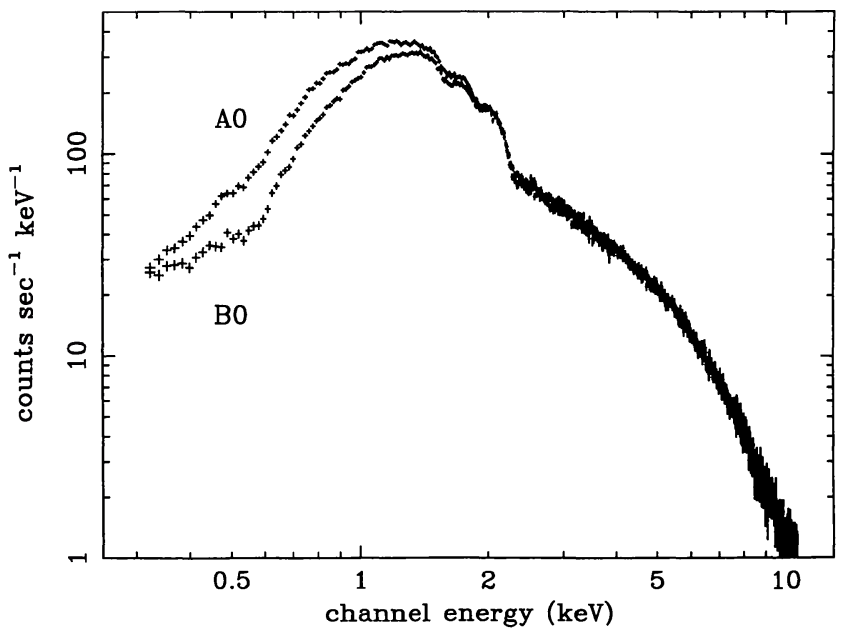

FIG. 8.-Comparison between the A and B detector central pixels for the on-axis Crab observation.

The strongest feature in the Crab data is the gold M-edge complex due to absorption of X-rays by the mirrors. The energy of the main gold edge in the A system appears at the expected value of $2.22 \mathrm{keV}$ (Figs. 8 and 9). However, the main edge appears at a higher energy of $2.25 \mathrm{keV}$ in the B system, which indicates a difference between the A and B mirrors. It is not unreasonable that the energies of gold edges from the $\mathrm{A}$ and B mirrors differ slightly. In fact, experiments to study Xray absorption fine structure allow the energy of the main absorption edge to be a free parameter (Lee et al. 1981). This is because there is an intrinsic uncertainty in determining the threshold energy of an absorption edge due to variations in the density of states near the Fermi energy that causes structure to be present within $\pm 10 \mathrm{eV}$ of $E_{0}$. Also, chemical-bonding effects can shift the apparent edge toward higher energies by up to 15 eV (Agarwal 1991; Lee et al. 1981).

To accurately fit the Crab between 0.5 and $0.8 \mathrm{keV}$ it was necessary to include features in the effective area that can be interpreted physically as gold $\mathrm{N}$ edges at energies of 0.546 , 0.643 , and $0.762 \mathrm{keV}$. The best-fit values for the absorption depths of the edges for each mirror-plus-detector combination were obtained using central-pixel Crab data along with the best-fit detector absorption parameters ( see $\S 3.7$ and Table 5). The absorption depths of the edges are as follows: $\tau=0.3,0.18$, and 0.12 , respectively, for $\mathrm{A}$ and $0.66,0.12$, and 0.16 for $\mathrm{B}$, with a typical error of 0.04 . The relative edge depths are roughly what we might expect to observe for the A system, and the depths of the 0.643 and $0.762 \mathrm{keV}$ edges in the B system are not significantly different from those in A. However, the $0.546 \mathrm{keV}$ edge depth in $\mathrm{B}$ is twice that in $\mathrm{A}$. The stronger "edge" in B can clearly be seen in the Crab data (Fig. 8); however, the absorption depth we measure may not be physical because of the various uncertainties in adjusting the effective area curve for B at low energies ( $\$ 3.7)$.

Two vignetting corrections are currently included in the standard BBXRT response matrix. First, the effective area curve is normalized by $62 \%$ under the assumption that one is analyzing data from the $\mathrm{A} 0$ pixel for an on-axis source. This correction factor accounts for $38 \%$ of the source counts being lost, primarily to the mask and the outer pixels ( $§ 2.2)$, and provides accurate fluxes for sources that are more or less centered in A0 ( $\$ 4)$. Second, for off-axis sources, energy-dependent vignetting corrections to the effective area are included in the response; these were obtained by modeling the throughput of the mirrors as a function of off-axis angle using ray-tracing techniques (Petre \& Serlemitsos 1985). The results of the ray tracing for off-axis observations are presented in Figure 10, which shows the ratio of off-axis to on-axis effective areas as a function of energy for various off-axis angles. The current raytracing calculations have been performed for point sources only.

A third correction that has not been included in the response matrix accounts for the (often significant) loss of photons for sources observed off axis due to the presence of the masks between the detector elements. If a source happens to sit on a mask, up to $50 \%$ of the photons reaching the focal plane can be lost. As an illustration of the potential severity of the problem, the distribution of photons in the focal plane for a point source observed in three different locations is shown in Figure 11. Here, the azimuthal angle $\Phi$ is arbitrarily measured with respect to the edge of the outer pixel nearest the source position and increases toward the center of that pixel (Fig. 11, top left). Due to the symmetry of the outer pixels, $\Phi$ ranges from $0^{\circ}$ to $45^{\circ}$. Case $a$ illustrates the distribution of photons for a point source that is directly on axis. For a source that is located $4^{\prime}$ off axis directly on the mask ( case $b$ ), the total collection efficiency is cut by half. However, in case $c$, where the source is off axis but centered in an outer pixel $\left(\Phi=45^{\circ} .0\right)$ the total collection efficiency is higher than in case $a$ due to the larger size of the outer pixels. We have used ray-tracing techniques to calculate the fraction of counts lost to the mask for a multitude of source positions. Figure 12 provides the flux-correction factor to be used for off-axis observations for sources having off-axis angles

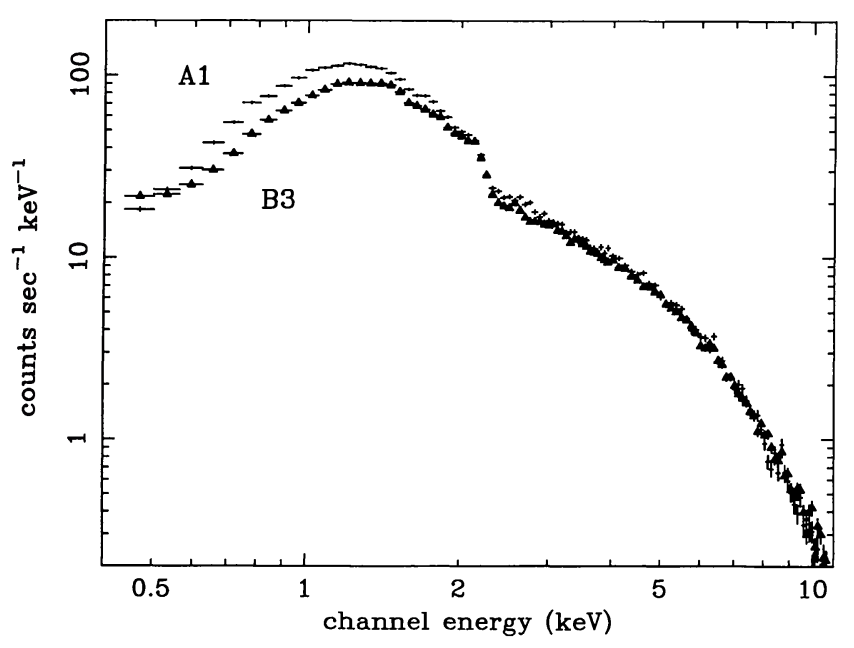

FIG. 9.-Comparison between the A1 and B3 outer pixels when the Crab Nebula is roughly centered in the detector. The B3 data are designated with triangles. 


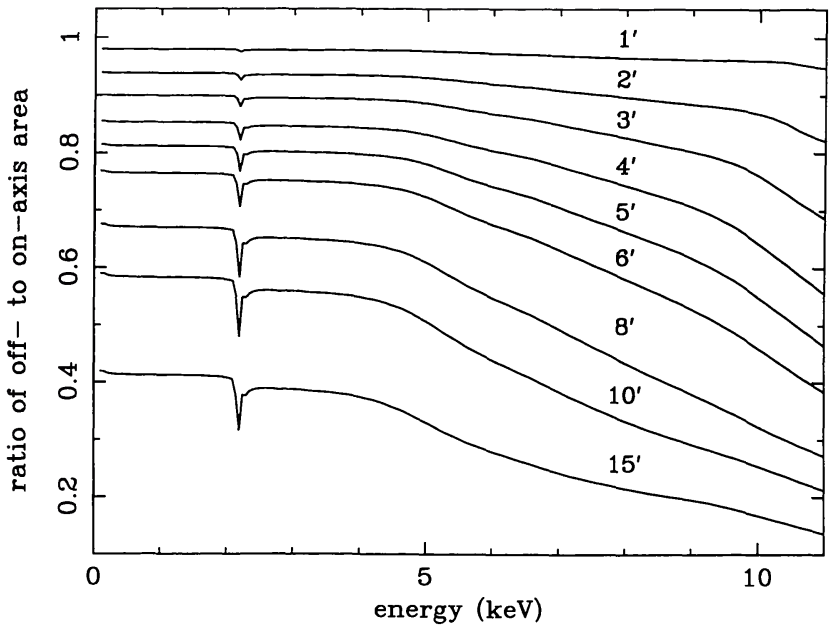

FIG. 10.-Ratio of off-axis to on-axis effective area as a function of energy for various off-axis angles (in arcminutes).

( $\Theta$ ) ranging from $0^{\prime}$ to $8^{\prime}$ and azimuthal angles of $\Phi=0.0$, $22^{\circ} .5$, and $45^{\circ}$.

To determine the flux for an off-axis point source, $\Theta$ and $\Phi$ must be known. If an aspect solution is available, the R.A. and decl. offsets and the roll angle (Table 1) provide both $\Theta$ and $\Phi$. Otherwise, $\theta$ and $\Phi$ are estimated by utilizing the available raytracing program. Once $\Theta$ and $\Phi$ are determined, Figure 12 gives the appropriate correction to the fluxes derived with the current BBXRT response matrix. For example, for a point source having $\Theta=5^{\prime}$ and $\Phi=45^{\circ}$, the flux correction factor for the A detector pixel in which the source is located (the "source pixel"; labeled as "pixel 1" in Figs. 11 and 12) is 1.23. The flux correction factor for each of the adjoining or "nonsource" outer pixels (labeled as "pixel 2" and "pixel 4" in Figs. 11 and 12 ) is $\sim 0.02$. To derive the flux of the source, divide the apparent flux (found from the spectral fit) by the flux correction factor for that pixel.

\subsection{The Detector Efficiency}

The detector efficiency was originally calculated including the values for Parylene $N, \mathrm{Al}, \mathrm{Si}$, and $\mathrm{Au}$ that had been specified to the manufacturers for the parylene and aluminum in the UV filter, the silicon dead layer in the detector, and the gold in the surface contacts (listed in Table 5). However, using these values in the response matrix provided a poor fit to the Crab, giving a reduced $\chi^{2}$ of about 2.5 in A0. Therefore, the current $\mathrm{A} 0$ and $\mathrm{B} 0$ pixel effective area curves were generated by allowing the optical depths of the various sources of absorption within and above the detectors to be free parameters in the fits to the Crab. Details of the procedure are given by Weaver (1993). For both detectors, we assumed that gold and aluminum absorption was uniform across the detector, while the parylene and silicon absorption was allowed to vary for each pixel. Although the Crab Nebula was not observed in all pixels, other bright sources that were observed at least twice, both on and off axis, were available for calibration of the outer pixels. The targets used to calibrate the outer pixels and to provide consistency checks between pixels are listed in Table 6 . The effective area curves of the outer A pixels were calculated by fitting spectra from each pixel simultaneously with an A0 spectrum of the same source and varying the effective area to give the best fit. A similar but more rigorous procedure was adopted for the outer B pixels because of the known uncertainty in the partial charge modeling. The magnitude of the partial charge was initially fixed by examining the shape of the low-energy side of the oxygen fluorescence airglow line. The effective area curves were then calculated by fitting spectra from each pixel simultaneously with a B0 spectrum of the same source and varying the effective area to give the best fit.

Some of the best-fit absorption values turn out to be unphysical (e.g., negative silicon) and so they will be discussed as "absorption parameters," which allow an improved fit to the Crab data. The best-fit detector absorption parameters for each pixel are given in Table 5 . The values in columns (2)-(7) are almost identical for all of the A pixels, while there is much less consistency between the best-fit values for the B pixels. The B detector requires a partial covering of gold described by

$$
\text { abs }=\exp \left(-\operatorname{Gold} 1 z-\operatorname{Gold} 2 z^{2}\right),
$$
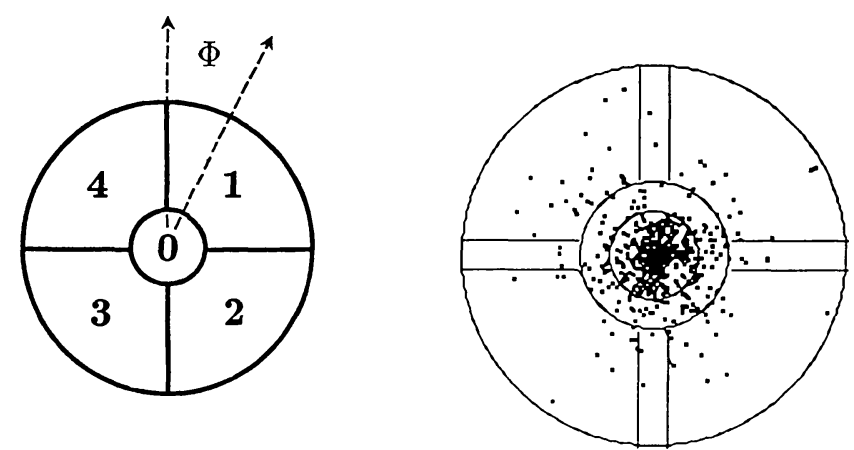

(a) $\Theta=0, \Phi=0$

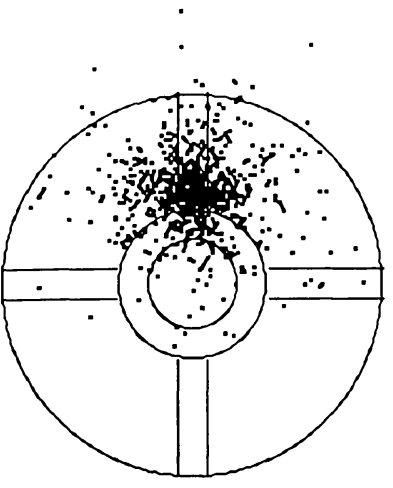

(b) $\Theta=4, \Phi=0$

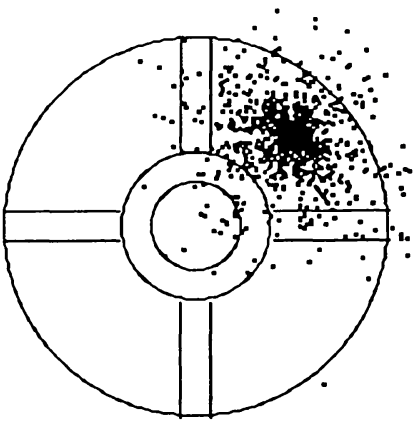

(c) $\Theta=6, \Phi=45$
FIG. 11.-Distribution of photons in the focal plane for a point source observed at different positions: ( $a$ ) on axis (in pixel $0 ; \theta=0.0, \Phi=0.0$ ), (b) $4^{\prime}$ off axis sitting directly on the mask ( $\Phi=0.0$; between pixels 1 and 4 ), and $(c)$ centered in an outer pixel ( pixel $1 ; \theta=6.0, \Phi=45^{\circ} .0$ ). In the upper left corner is a schematic diagram of the A detector; the pixels are arbitrarily numbered for purposes of cross-referencing this figure with Fig. 12. The pixel numbers assigned here should not be confused with the true designation of pixels as $\mathrm{A} 0, \mathrm{~A} 1, \mathrm{~A} 2, \mathrm{~A} 3$, and $\mathrm{A} 4$. 

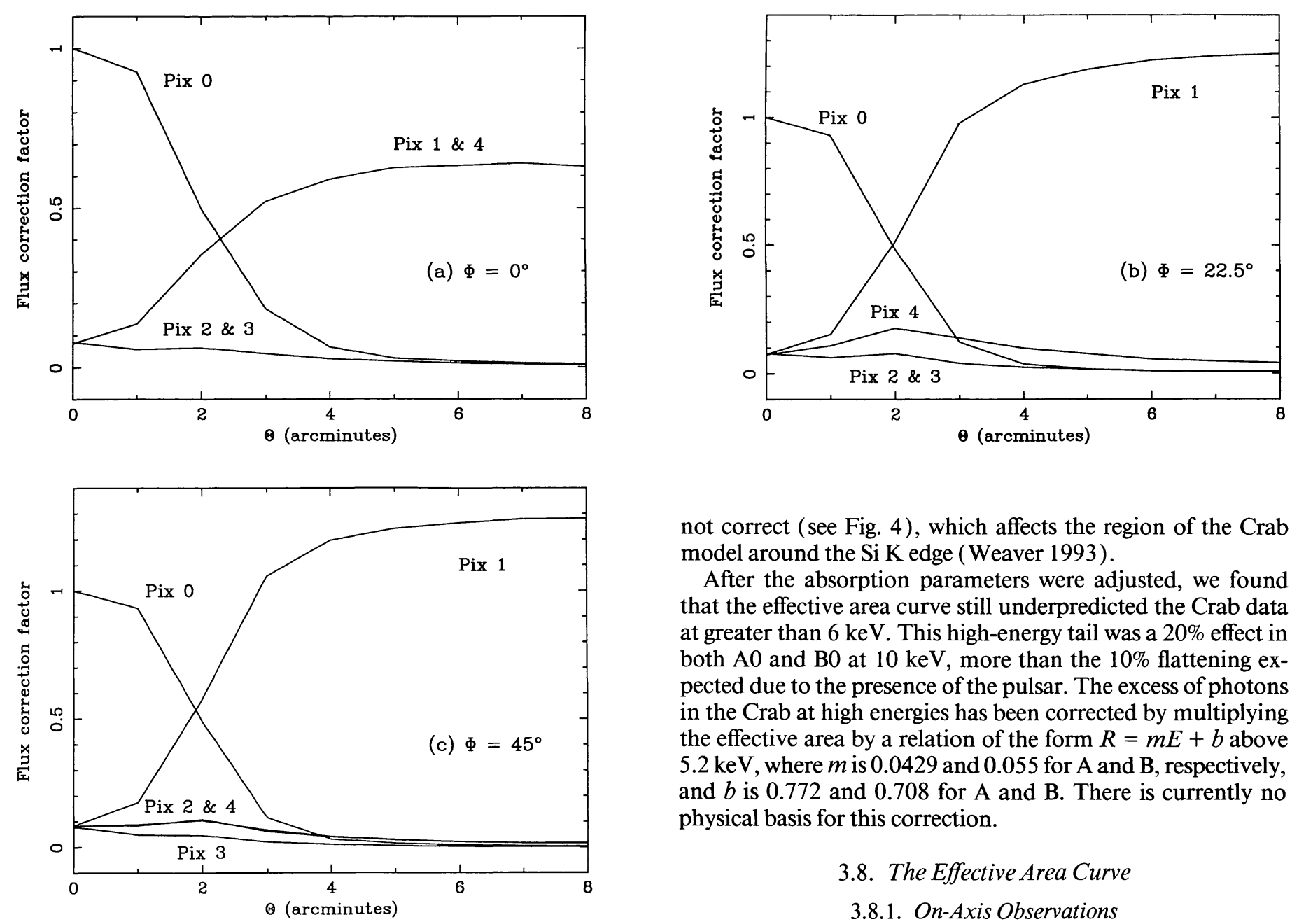

FIG. 12.-Flux-correction factor as a function of off-axis angle for three different azimuthal angles: $(a) \Phi=0^{\circ},(b) \Phi=22^{\circ} .5$, and $(c) \Phi=45^{\circ}$. The pixel numbers correspond to those in the upper left panel in Fig. 11, where the "source" pixel is typically pixel 1 , pixel 0 is the central pixel, and pixels 2 and 3 are generally farthest from the source position.

where Gold1 is the number of clumps times thickness, Gold2 is the number of clumps times thickness squared, and $z$ is defined as the energy-dependent absorption cross section. This is not a physical model, but is merely a way of adding a free parameter.

The negative values that we measured for the thickness of the Si dead layer (Table 5, col. [5]) are physically meaningless. This implies that we do not understand one or more of the following: (1) the effects of the partial charge collection around the silicon edge, (2) the thickness of the silicon dead layer in the detector, (3) the importance of the low-energy partial charge wing discussed earlier ( $\S 3.3)$, or (4) the silicon-absorbing column to the Crab. Negative silicon also affects the bestfit parylene absorption, causing it to be too high, because it compensates (at low energies) for the decreased silicon. The "silicon problem" is worse in the B detector. However, this primarily occurs because the present partial charge model is

not correct (see Fig. 4), which affects the region of the Crab model around the Si K edge (Weaver 1993).

After the absorption parameters were adjusted, we found that the effective area curve still underpredicted the Crab data at greater than $6 \mathrm{keV}$. This high-energy tail was a $20 \%$ effect in both $\mathrm{A} 0$ and $\mathrm{B} 0$ at $10 \mathrm{keV}$, more than the $10 \%$ flattening expected due to the presence of the pulsar. The excess of photons in the Crab at high energies has been corrected by multiplying the effective area by a relation of the form $R=m E+b$ above $5.2 \mathrm{keV}$, where $m$ is 0.0429 and 0.055 for A and $\mathrm{B}$, respectively, and $b$ is 0.772 and 0.708 for $\mathrm{A}$ and $\mathrm{B}$. There is currently no physical basis for this correction.

\subsection{The Effective Area Curve}

\subsubsection{On-Axis Observations}

Figure 13 shows the current on-axis mirror-plus-detector effective area curves for A0 and B0. Figure $14 a$ illustrates the best power-law fit to the on-axis A0 Crab observation ( 580 counts $\mathrm{s}^{-1}$ in $\mathrm{A} 0$; data from 3.0569-3.068 days MET), which results in $\Gamma=2.1$ and $N_{\mathrm{H}}=3 \times 10^{21} \mathrm{~cm}^{-2}$. The residuals from the fit are shown in Figure 14b. Figure $15 a$ shows the best power-law fit to the on-axis B0 Crab observation (from 3.0569-3.068 days MET), which also results in $\Gamma=2.1$ and $N_{\mathrm{H}}=3 \times 10^{21} \mathrm{~cm}^{-2}$. The residuals from the fit are shown in Figure $15 \mathrm{~b}$. At the present time, the effective area is understood at the $5 \%$ level in A0 with up to $10 \%$ residuals at very low energies $(<0.6 \mathrm{keV})$. The area is also understood at the $5 \%-10 \%$ level in B0 over 0.6-9 keV. However, residuals to the Crab in B0 appear both below $0.6 \mathrm{keV}$ and above $9 \mathrm{keV}$ at the $10 \%-$ $15 \%$ level. The $\sim 10 \%$ residuals above $9 \mathrm{keV}$ appear to be caused by incomplete modeling of the $8.3 \mathrm{keV}$ nickel edge. There also appears to be a larger mismatch to the $\mathrm{Al}, \mathrm{Si}$, and $\mathrm{Au}$ edges in $\mathrm{B} 0$ than in $\mathrm{A} 0$, and there is a narrow feature present at $1.38 \mathrm{keV}$ in $\mathrm{B} 0$. This feature is at worst a $10 \%$ residual, and less than $10 \mathrm{eV}$ equivalent width when fit with a Gaussian. In addition, a less than 5\% "bump" in the residuals still exists around $7.5 \mathrm{keV}$ in both $\mathrm{A}$ and $\mathrm{B}$.

The worst residuals in both detectors are at $\sim 0.5 \mathrm{keV}$. When the on-axis Crab data are split into night (3.057-3.062 days 
TABLE 5

BEST-FIT DETECTOR ABSORPTION THICKNESS

\begin{tabular}{|c|c|c|c|c|c|c|}
\hline $\begin{array}{l}\text { Detector } \\
\text { (1) }\end{array}$ & $\begin{array}{c}\text { Step }^{a} \\
(2)\end{array}$ & $\begin{array}{l}\text { Parylene } \\
\text { (A) } \\
\text { (3) }\end{array}$ & $\begin{array}{l}\text { Aluminum } \\
(\AA) \\
(4)\end{array}$ & $\begin{array}{l}\text { Silicon } \\
(\AA) \\
(5)\end{array}$ & $\begin{array}{l}\text { Gold1 } 1 \\
\text { (A) } \\
(6)\end{array}$ & $\begin{array}{c}\text { Gold2 } \\
(\AA) \\
(7)\end{array}$ \\
\hline Specification ....... & & 4000 & 1500 & 900 & 200 & \\
\hline A0 & $3.40 \mathrm{E}-05$ & 8150 & 1625 & -100 & 126 & 0.0 \\
\hline A1 & $3.40 \mathrm{E}-05$ & 8150 & 1625 & -100 & 126 & 0.0 \\
\hline A2 & $3.40 \mathrm{E}-05$ & 8150 & 1625 & -100 & 126 & 0.0 \\
\hline A3 & $3.40 \mathrm{E}-05$ & 8150 & 1625 & -100 & 126 & 0.0 \\
\hline A4 & $7.50 \mathrm{E}-05$ & 8150 & 1625 & -100 & 126 & 0.0 \\
\hline 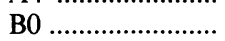 & $4.40 \mathrm{E}-05$ & 11000 & 1900 & -1000 & 212 & 44944 \\
\hline B1 .......................... & $6.00 \mathrm{E}-05$ & 4400 & 1900 & -2650 & 212 & 44944 \\
\hline B2 & $9.00 \mathrm{E}-05$ & 5500 & 1900 & -2000 & 212 & 44944 \\
\hline 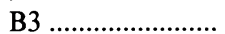 & $9.00 \mathrm{E}-05$ & 11000 & 1900 & -3200 & 212 & 44944 \\
\hline 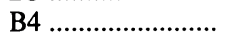 & $7.00 \mathrm{E}-05$ & 8250 & 1990 & -3300 & 212 & 44944 \\
\hline
\end{tabular}

${ }^{\text {a }}$ Step refers to $S(E)$, the amount of partial change (eq. [3]) included in the response function.

MET) and day (3.062-3.068 days MET) there is an indication that the low-energy residuals change with time, ranging from a $5 \%$ to a $15 \%$ effect. In B0, they are almost absent in the background-subtracted daytime data (Fig. 16a) but are strong in the nighttime data (Fig. 16b). This effect is reversed in the A0 detector, with the residuals being stronger in the daytime data. There is no correlation between the size of the residuals and the detector guard rate $(\S 5.1)$, the rate of light-emitting diode (LED) firings ( $\S 5.1)$, the Earth angle $(\S 5)$, or background subtraction. An alternative explanation would be that the gain or resolution at low energies may have changed with time. The magnitude of the residuals can be measured as follows: fitting the positive $\sim 0.5 \mathrm{keV}$ feature with a narrow Gaussian ( $E=$ $0.41 \mathrm{keV}$ ) gives an equivalent width of less than $140 \mathrm{eV}$ in A0 and less than $220 \mathrm{eV}$ in B0. Fitting the negative feature gives an energy of $0.52 \mathrm{keV}$ (a slightly lower energy than an oxygen edge at $0.53 \mathrm{keV}$ ). Fitting with a notch (width $0.03 \mathrm{keV}$ ) gives a covering fraction for the notch of less than 0.4 for $\mathrm{A} 0$ and less than 0.65 for B0.

Figure 17 shows the confidence contours for $N_{\mathrm{H}}$ versus $\Gamma$ derived from all 10 pixels when the Crab is approximately on axis. First, it can be seen that the $90 \%$ confidence contours for $\mathrm{A} 0$ and $\mathrm{B} 0$ overlap, indicating that the two central pixels (the source pixels) give consistent results for the on-axis Crab observation. Second, this figure illustrates that for the outer pixels (the nonsource pixels), the systematic uncertainties that remain $(\Delta \Gamma=0.2)$ are 2-3 times greater than the statistical uncertainties in fitting the Crab. This systematic uncertainty measured in the outer pixels may be caused by fact that we have

TABLE 6

OUTER-PIXEL CALIBRATION SOURCES

\begin{tabular}{cccc}
\hline \hline Pixels & Primary & Secondary & Consistency check \\
\hline A1 and B3 & Crab & PKS 2155-304 & Perseus \\
A2 and B4 & Perseus & Capella & PKS 2155-304 \\
A3 and B1 & Cygnus X-2 & Perseus & PKS 2155-304 \\
A4 and B2 & Cygnus X-1 & Perseus & Earthglow \\
\hline
\end{tabular}

not correctly accounted for the energy dependence in the mirror PSF ( $§ 3.8 .3$ below).

\subsubsection{Off-Axis Observations (Source Pixel)}

Table 7 lists the lowest available channels and the lowest reliable channels for all pixels, along with the corresponding channel energies. A0, B0, and the outer A pixels ( A1, A2, and A3) are reliable down to their lowest usable energies, determined by the setting of the detector noise threshold. The A4 pixel is particularly noisy and so is only reliable down to about $0.7 \mathrm{keV}$. Also, because of the large correction and uncertainty in modeling the partial charge, the outer B pixels are reliable only down to $0.5-0.7 \mathrm{keV}$. The less than $0.7 \mathrm{keV}$ excess seen in the Crab data in the outer B pixels (Fig. 9) has not been removed because it is not possible with our present modeling of the partial charge with a step function. A full understanding of the magnitude and shape of the partial charge is needed to utilize the outer B pixels at low energies.

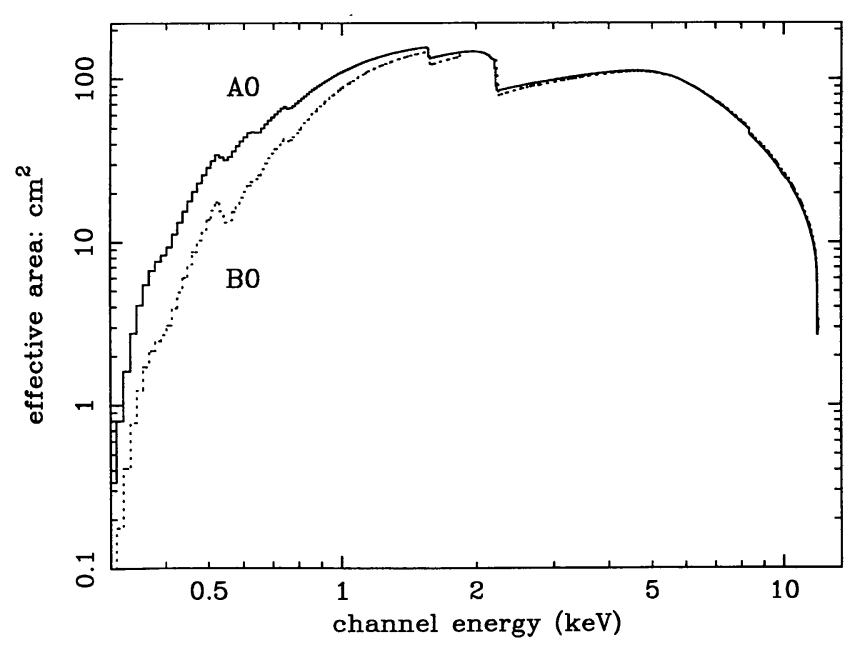

FIG. 13.-The on-axis mirror-plus-detector A0 and B0 effective area curves. 


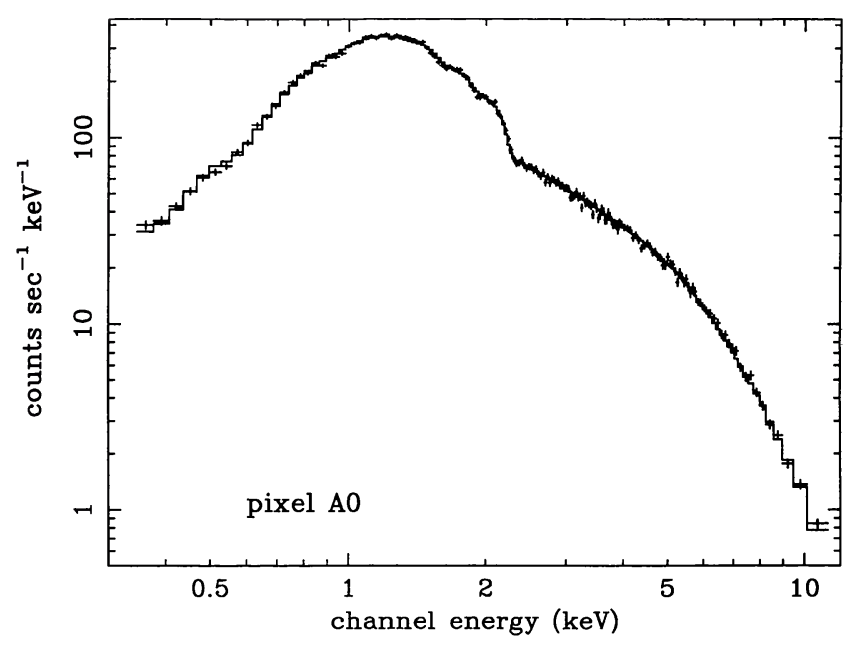

FIG. $14 a$

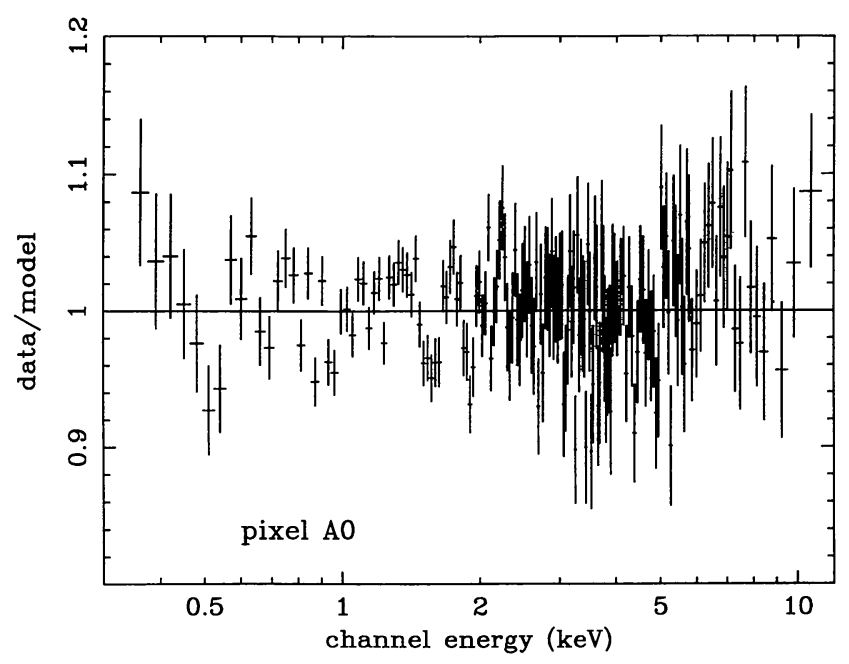

FIG. $14 b$

FIG. 14.-(a) Plot of the on-axis A0 Crab data and best-fit model: $\Gamma=2.115, N_{\mathrm{H}}=3.04 \times 10^{21} \mathrm{~cm}^{-2}$. For this fit, $\chi_{\nu}^{2}=1.4$. (b) Ratio of the data to the model.

The confidence contours for $N_{\mathrm{H}}$ versus $\Gamma$ for all pixels when the Crab is 3.0 off axis are shown in Figure 18. In this case, the source pixels are $\mathrm{A} 1$ and $\mathrm{B} 3$. The index measured for $\mathrm{A} 1(\Gamma=$ 2.16 ) is steep and is inconsistent with the index measured for B3 $(\Gamma=2.09)$. This represents the largest discrepancy still remaining between pairs of source pixels $(\Delta \Gamma \sim 0.10)$, although this should be an observable problem only for bright sources $\left(\gtrsim 10\right.$ counts $\mathrm{s}^{-1}$ ) with good statistics. The remaining discrepancy between A1 and B3 is smaller than the general systematic uncertainties observed between outer, nonsource pixels when the Crab is on axis (Fig. 17).

\subsubsection{On- and Off-Axis Observations (Nonsource Pixels)}

For a point source observed with BBXRT, there are still large systematic residuals when attempting to use data that are

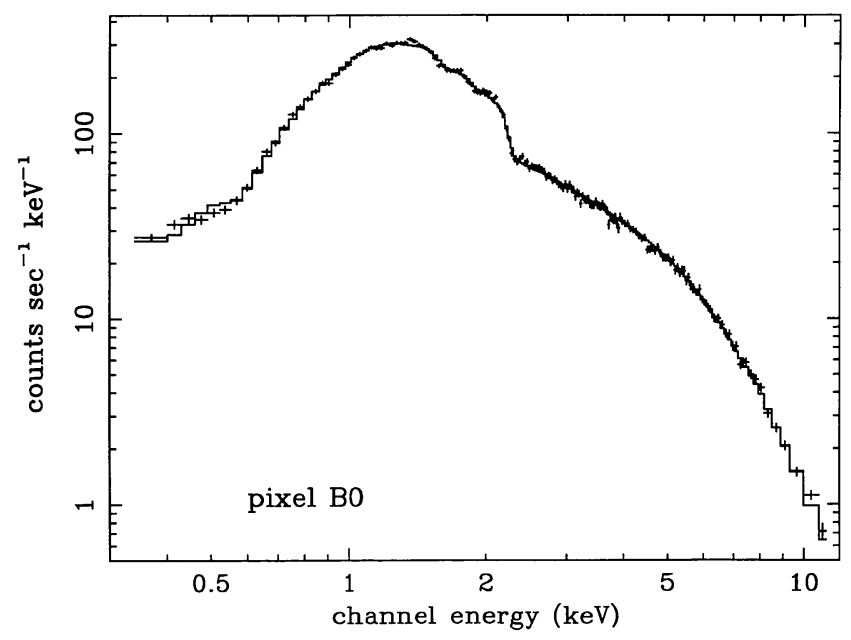

FIG. $15 a$ not from the source pixel (i.e., from a nonsource pixel). During the on-axis observation, the Crab was actually offset by about 1 ' toward A3 and B1 (§3.4). From Figure 17, it can be seen that for this observation, for 5 out of 6 of the pixels other than $\mathrm{A} 0, \mathrm{~B} 0, \mathrm{~A} 3$, and $\mathrm{B} 1$ (i.e., those pixels in which the majority of the Crab flux is coming from a region greater than $5^{\prime}$ away from the core of the PSF), the spectra tend to be flatter. The effect is even more obvious during the off-axis Crab observation (Fig. 18). In this case, the source pixels are $\mathrm{A} 1 / \mathrm{B} 3$ and $\mathrm{A} 0 / \mathrm{B} 0$, while A3/B1 are the furthest from the source in detector coordinates. Clearly, the measured photon indices become much flatter with increasing angular distance from the Crab.

The flattening in the Crab spectrum observed in nonsource pixels is due to the increased presence of a high-energy tail when we examine data obtained from a greater than $5^{\prime}-6^{\prime}$ radius away from the center of the source in detector coordinates.

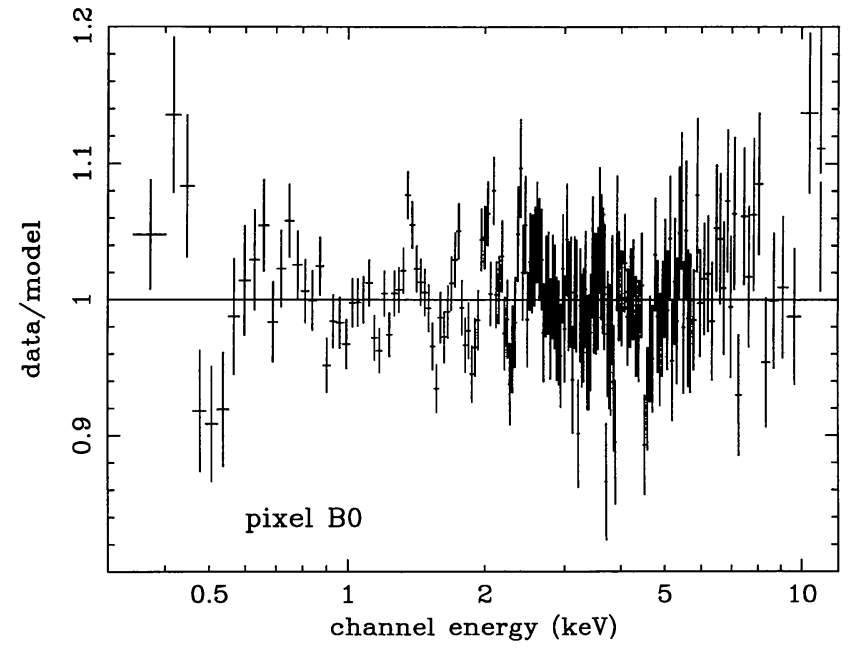

FIG. $15 b$

FIG. 15.- (a) Plot of the on-axis B0 Crab data and best-fit model: $\Gamma=2.12, N_{\mathrm{H}}=3.08 \times 10^{21} \mathrm{~cm}^{-2}$. For this fit, $\chi_{\nu}^{2}=1.38$. $(b)$ Ratio of the data to the model. 

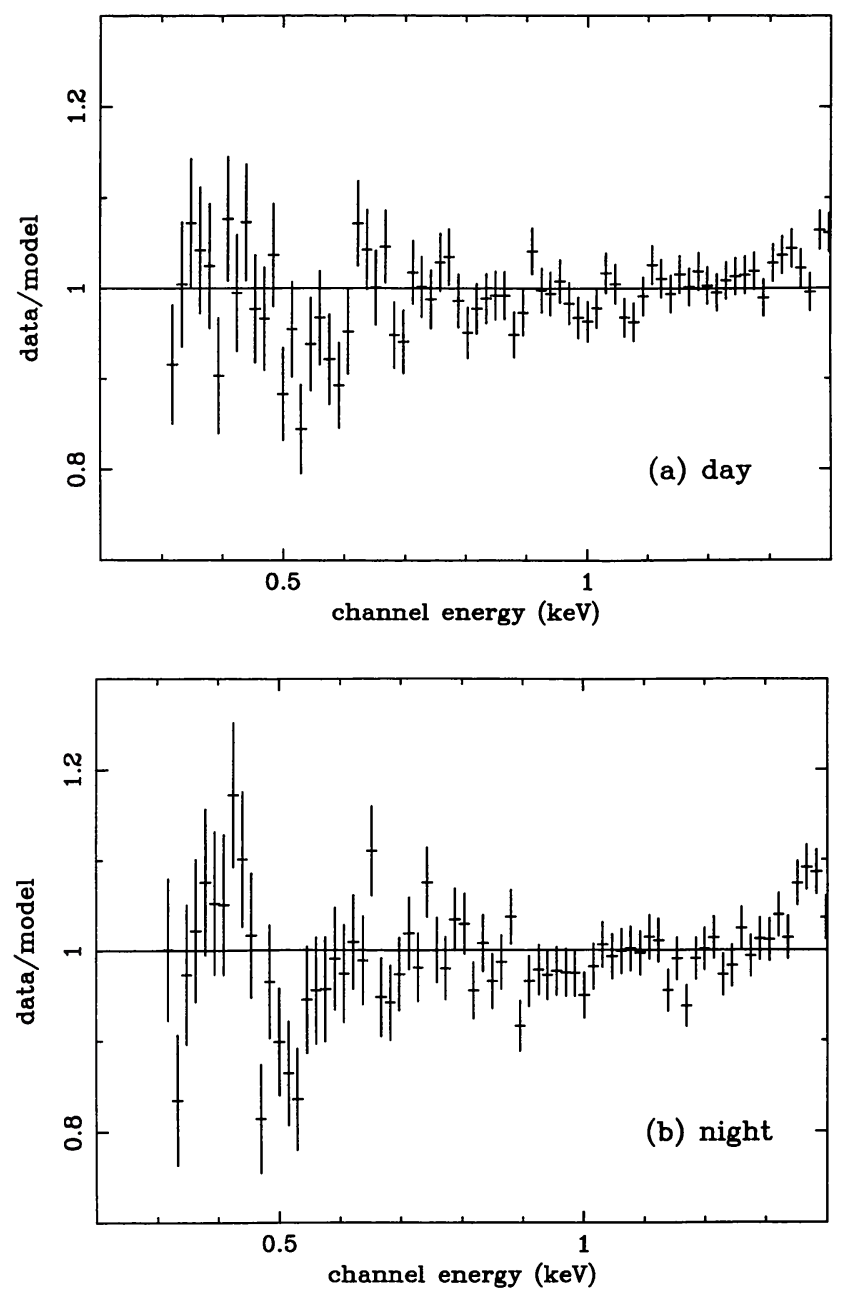

FIG. 16.-Plot of the $0.3-1.4 \mathrm{keV}$ ratio of the unbinned B0 Crab spectra to the best-fit power-law model for the on-axis observation divided into $(a)$ day and $(b)$ night.

This flattening can be seen in power-law fits to data from the outer pixels when the Crab is observed approximately on axis. Figure 19 shows a plot of the ratio of the A2 and B4 Crab spectra to an absorbed power-law model having an index of $\Gamma=$ 2.1. In this case, the best-fit $N_{\mathrm{H}}$ is the "expected" value of $3 \times$ $10^{21} \mathrm{~cm}^{-2}$. A tail at high energies (which is a $20 \%$ effect) is observed in the ratio of the data to the model.

One explanation for the high-energy tail seen in the nonsource pixels is an energy dependence of the mirror point-response function for large radii, in the sense that higher energy $\mathrm{X}$-rays are scattered into larger angles. Although measurements appropriate to test for an energy dependence are not available for the BBXRT mirrors, this effect has been observed for similar mirrors which are presently flying on $A S C A(\mathrm{H}$. Kunieda and the $A S C A$ calibration team 1993, private communication). Preliminary measurements of the $A S C A$ mirrors indicate that $7 \%$ of the flux at $2 \mathrm{keV}, 15 \%$ of the flux at $5 \mathrm{keV}$, and $17 \%$ of the flux at $8 \mathrm{keV}$ is scattered from the core of the mirror PSF into a greater than 6 ' radius (thus extending the wings of the PSF for higher energies). This energy-depen-

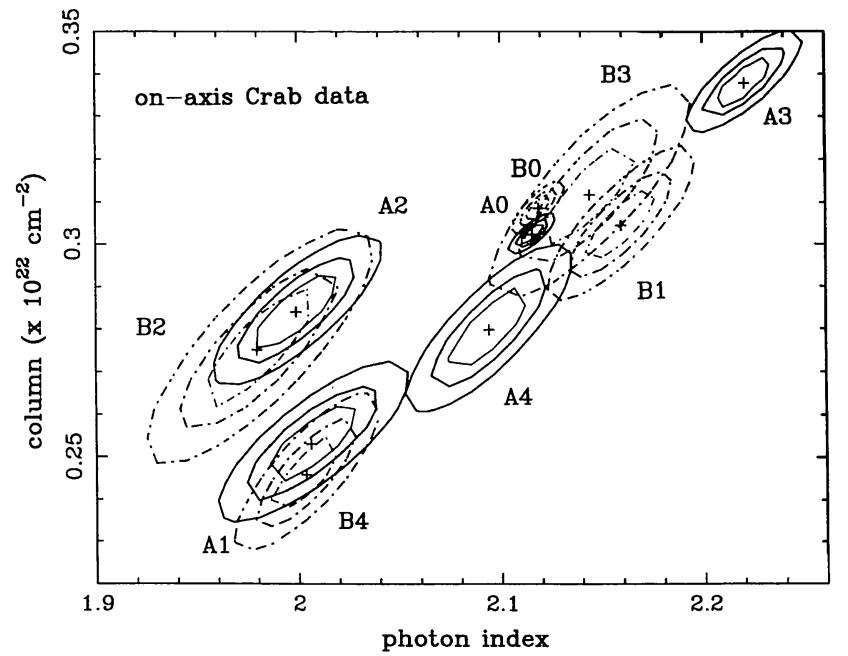

FIG. 17.-Confidence contours for $N_{\mathrm{H}}$ vs. $\Gamma$ for all pixels when the Crab is on axis. The contours shown are $68 \%, 90 \%$, and $99 \%$. Contours for the A pixels are solid lines.

dent scattering, due to the residual roughness of the mirror foils, has exactly the right magnitude to explain the high-energy tail observed in the outer-pixel BBXRT data when the Crab is centered (Fig. 19), although off-axis measurements of the Crab indicate that this tail is an even larger effect than expected for a simple energy dependence in the PSF.

The BBXRT mirror effective area curve was generated for the entire focal plane. However, we used only the A0 data (essentially from the beam core) to modify the effective area to fit the Crab. If scattering out of the beam core is energy dependent (which is highly probable given the $A S C A$ results), then a larger fraction of high-energy photons than low-energy photons are scattered out of the core. This means that the measured slope of the Crab when using only central pixel data should be "too steep." We may have inadvertently corrected for this steepening by altering the mirror gold density fraction from 0.87 to 0.84 (although the original spectral steepening was less than a 5\% effect, see $\S 3.6$ ). It is therefore likely that our calibration contains a systematic error in the sense that measured spectral slopes will be too flat by about $5 \%-10 \%$ (in

TABLE 7

RELIABILITY OF LOWEST ENERGY EFFECTIVE AREA

\begin{tabular}{|c|c|c|c|c|}
\hline Pixel & $\begin{array}{l}\text { Lowest Usable } \\
\text { Channel }\end{array}$ & $\begin{array}{c}\text { Energy } \\
(\mathrm{keV})\end{array}$ & $\begin{array}{l}\text { Lowest Reliable } \\
\text { Channel }\end{array}$ & $\begin{array}{c}\text { Energy } \\
(\mathrm{keV})\end{array}$ \\
\hline A0 ....... & 16 & 0.29 & 16 & 0.29 \\
\hline Al ................ & 26 & 0.41 & 26 & 0.41 \\
\hline A2 ........ & 26 & 0.42 & 26 & 0.42 \\
\hline A3 ....... & 27 & 0.44 & 27 & 0.44 \\
\hline A4 ............. & 25 & 0.41 & 43 & 0.70 \\
\hline B0 ............ & 17 & 0.30 & 17 & 0.30 \\
\hline B1 ............ & 27 & 0.42 & 39 & 0.60 \\
\hline B2 $\ldots \ldots$ & 28 & 0.43 & 34 & 0.52 \\
\hline B3 $\ldots . . .$. & 28 & 0.45 & 34 & 0.54 \\
\hline 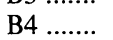 & 29 & 0.44 & 45 & 0.69 \\
\hline
\end{tabular}




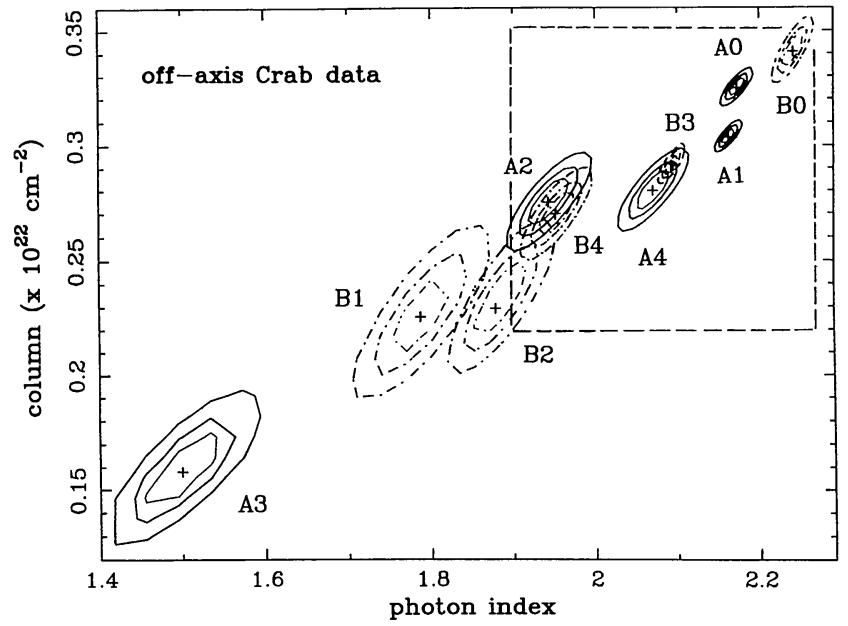

FIG. 18.-Confidence contours for $N_{\mathbf{H}}$ vs. $\Gamma$ for all pixels when the Crab is 3. 0 off axis. The box delineates the region shown in Fig. 17. The contours shown are $68 \%, 90 \%$, and $99 \%$. Contours for the A pixels are solid lines.

$\Delta \Gamma$ ) when using data from a source pixel, whether the source is observed on or off axis. However, this small effect should only be noticeable for sources having count rates greater than $\sim 10$ counts s $^{-1}$. For data from a nonsource pixel, the systematic flattening at high energies, thought to be caused by the energy dependence in the PSF, can easily dominate statistical uncertainties for most point sources (Figs. 18 and 19).

\section{FLUX CALIBRATION}

To determine the normalization of the BBXRT effective area curve using the Crab Nebula, corrections must be applied to the observed count rate because some of the X-ray events are not recorded in the telemetry. There are four reasons for this, two of which are dead-time effects. First, if an event occurs within $\sim 100 \mu$ s of a previous event in the same pixel, then it is lost and the earlier event is flagged. Second, the telemetry is only able to hold 252 events per frame ( 2016 events s$\left.^{-1}\right)$ and so once the buffer is full, additional events will be lost before the start of the next telemetry frame. Third, events in the same pixel which occur very close together (within $20 \mu \mathrm{s}$ ) may not be sensed as different events in the electronics. This causes a pile-up of pulse heights that can make multiple events appear as one event with a higher energy and may affect the spectrum for large count rates. Finally, events may be flagged with one of the quality flags: guard, large event, pixel-to-pixel, or LED (§ 5.1). When extracting spectra, we normally exclude any flagged events.

The effects of dead time (assumed to be energy independent), flagging, and pulse pile-up were examined using all of the events in the telemetry for a $1 \mathrm{~s}$ interval for the onaxis A0 Crab observation at 3.056 days $\left(3^{\mathrm{d}} 4839^{\mathrm{s}}\right)$. The observed count rate in the Crab spectrum is the true count rate corrected for dead-time effects $\left(c_{d}=0.855\right)$, the flagging ratio $\left(c_{f}=0.79\right)$, and the ratio of the instantaneous to the average count rate $\left(c_{\mathrm{av}}=0.96\right)$. This gives

$$
R_{\text {obs }}(\text { spectrum })=c_{d} c_{f} c_{\text {av }} R_{\text {true }}=0.648 R_{\text {true }} .
$$

An absorbed power-law fit to the on-axis Crab observation in A0 gives a normalization of $6.16 \pm 0.04$ photons $\mathrm{s}^{-1} \mathrm{keV}^{-1}$ $\mathrm{cm}^{-2}$ at $1 \mathrm{keV}$ (90\% confidence errors). After applying the above correction, this results in

$$
N(E)=9.51 E^{-2.11} e^{-\sigma(E) N_{\mathrm{H}}},
$$

where $N(E)$ is given in photons $\mathrm{s}^{-1} \mathrm{keV}^{-1} \mathrm{~cm}^{-2}$. The value of 9.51 that we find for the derived normalization at $1 \mathrm{keV}$ agrees well with the normalizations measured by previous experiments (Toor \& Seward 1974; Burrows 1982; Turner et al. 1989 ) and indicates that our derived Crab flux is accurate to within $\sim 15 \%$.

The flux calibration described above only applies to sources observed on axis in the A detector. Since we have not included the B detector offset in the effective area, only data from the A detector should be used to determine absolute source fluxes. We have also not included the effects of the mask in the effective area calculation. To account for the mask, one must apply a flux correction factor ( see $\S 3.6$ and Fig. 12).

\section{THE DETECTOR BACKGROUND}

There are four major contributions to the BBXRT detector background: internal background, particles, events from the cosmic or diffuse X-ray background (DXRB), and X-ray contamination at low energies from solar radiation scattered off Earth's atmosphere. This "Earthglow" is the dominant lowenergy component for daytime observations, and its intensity correlates with the "Earth angle," defined as the angle between the telescope pointing direction and the direction of the center of the Earth. During nighttime observations, the instrumental background and the DXRB contribute equally at low energies.

\subsection{Background Rejection Techniques}

Five anticoincidence techniques were used for BBXRT background rejection. Any of the following conditions caused

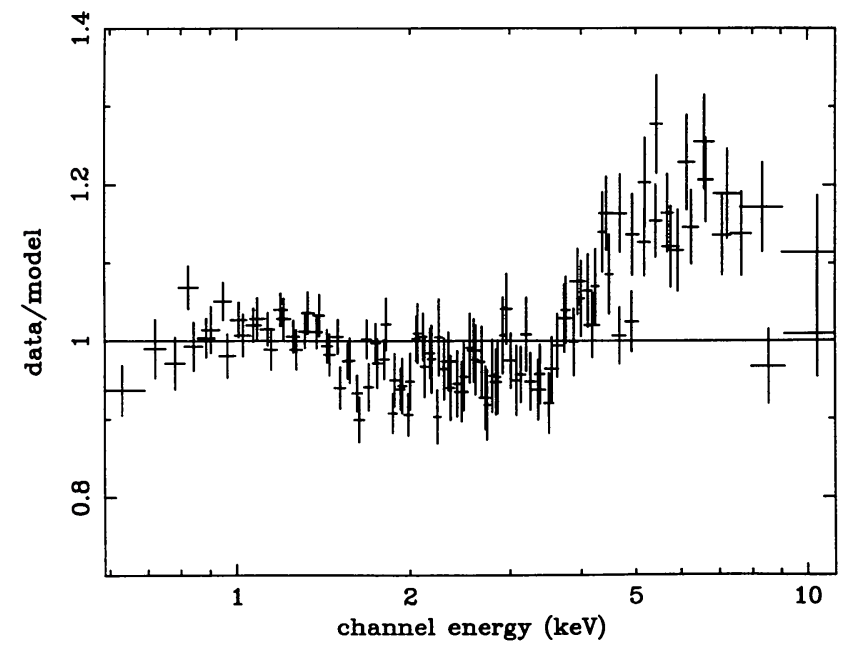

FIG. 19.- Ratio of Crab data from A2 and B4 to a power-law model when the Crab is centered in $\mathrm{A} 0$. In this case, $\Gamma$ is fixed to be 2.1 and the best-fit value of $N_{\mathrm{H}}$ is $3.0 \times 10^{21} \mathrm{~cm}^{-2}$. 
a detected event to be flagged as a "bad" event and not analyzed. First, pulse-pulse anticoincidence caused an event to be flagged when a simultaneous event $(<120 \mu \mathrm{s})$ was detected in the same pixel. Second, bad pulse heights due to splitting of events near pixel boundaries were measured by pixel-pixel anticoincidence, which worked mostly for large events $(\gg 10$ $\mathrm{keV}$ ). Third, very-large-event anticoincidence flagged an event that deposited a charge larger than that expected for a $14 \mathrm{keV}$ $\mathrm{X}$-ray. These often triggered pulses in adjacent pixels. Fourth, events were flagged when the plastic scintillator guard was triggered simultaneously $(<150 \mu \mathrm{s})$. A housekeeping parameter called the "guard rate" measured the rate of particle interactions with the guard. Finally, events were flagged when simultaneous firing of the LED opto-feedback circuits occurred $(<1 \mathrm{~ms})$.

\subsection{The Non-X-Ray Background}

The non-X-ray detector background is made up of electronic noise, microphonics, and particle events. Electronic noise is forced to be a small effect by setting the discriminator thresholds above $\sim 0.3-0.5 \mathrm{keV}$, the level at which it begins to contribute appreciably $(\S 2.1)$. Microphonics are measured by the use of accelerometer data and are different from the noise associated with the ordinary detector leakage current (responsible for $\sigma_{A}^{2}$ described in $\S 3.2$ ). Microphonics can be a major contribution at low energies (less than $0.8 \mathrm{keV}$ ). They are produced by changes in capacitance at the charge collection point of the preamplifier front-end junction field effect transistor when mechanical vibration of the rigid high-voltage lead within the cryostat is induced by external motions. Bursts of microphonics events typically last about $2 \mathrm{~ms}$, occur once every $10 \mathrm{~s}$, and contain up to 5 events spread over various pixels within a given detector. During data extraction, microphonics events can be eliminated for weak sources.

Particle events are the primary source of the non-X-ray background. At the end of the mission (8.3-8.8 days MET), several hours of data were obtained with the instrument covers closed to measure the non-X-ray background. During this time, the instrument was not passing through the South Atlantic Anomaly (SAA). Figure $20 a$ shows the co-added non-Xray background count rates with microphonics removed for the four outer A pixels versus time. The data are grouped into $200 \mathrm{~s}$ bins and range over channels $30-511(0.5-12 \mathrm{keV})$. A similar plot for the B detector outer pixels is shown in Figure $20 \mathrm{~b}$. The guard rate, which measures the rate of particle interactions with the plastic scintillator surrounding most of the detector, is plotted in Figure $20 c$. The guard rate is also averaged over $200 \mathrm{~s}$ bins and ranges from $\sim 800$ to 2000 per $8 \mathrm{~s}$ housekeeping frame, typical of periods outside the SAA. It can be seen that the non-X-ray background event rate clearly follows the guard rate.

The outer-pixel non-SAA count rates for the non-X-ray background are plotted against the associated guard rate in Figures $21 a$ and $21 b$. A correlation is clearly present, and it is approximately linear for guard rates greater than about 1000 and less than about 2000. This correlation allows the non-Xray component of the background to be easily predicted for a known guard rate. Below a guard rate of 1000 , the non-X-ray background reaches a lower threshold at about 0.15 and 0.18

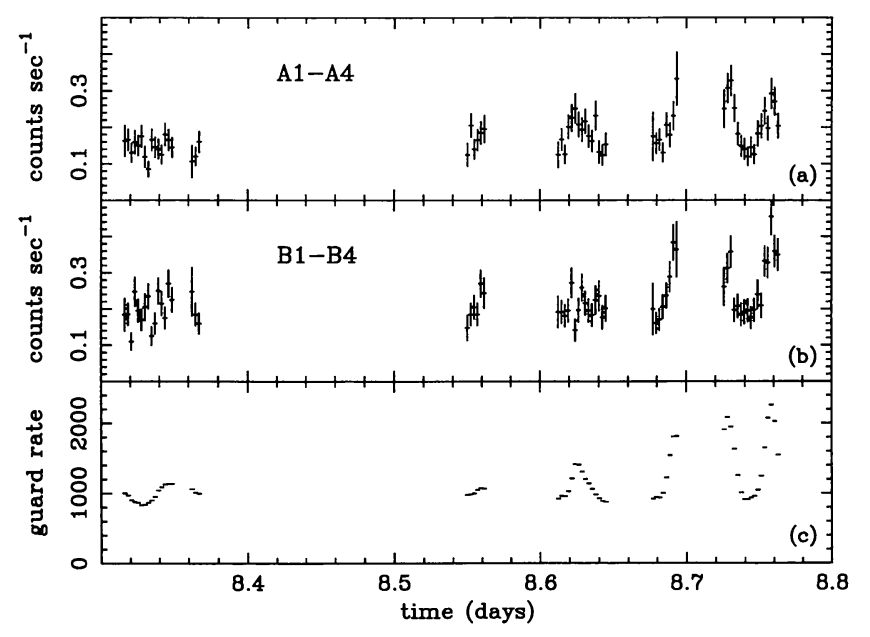

Fig. 20.-Non-X-ray background from closed-cover observations on day 8 of the mission as a function of time: $(a)$ count rate in channels 30 511 for the $\mathrm{A} 1-\mathrm{A} 4$ pixels, $(b)$ count rate in channels $30-511$ for the B1-B4 pixels, and $(c)$ detector A guard rate (defined in the text). The data have been accumulated in $200 \mathrm{~s}$ time bins.

counts $\mathrm{s}^{-1}$ in the summed outer A pixels and B pixels, respectively. The magnitude of the non-X-ray background (excluding microphonics) is $1.5 \times 10^{-3}$ counts $\mathrm{keV}^{-1} \mathrm{~s}^{-1}$ in each central pixel and $\sim 8 \times 10^{-3}$ counts $\mathrm{keV}^{-1} \mathrm{~s}^{-1}$ in each outer pixel, with the exception of A4 which has a very strong non-X-ray background component below $0.7 \mathrm{keV}$. The non$\mathrm{X}$-ray background in the central pixels is very flat between 0.4 and $11.0 \mathrm{keV}$ ( $\Gamma$ is approximately -0.2 ) and rises below 0.4 $\mathrm{keV}$. Illustrations of the slightly more complex shape of the non-X-ray background in the outer pixels can be found in Weaver (1993).

\subsection{The Diffuse X-Ray Background during Orbit Night}

For nighttime source observations and Earth angles greater than $90^{\circ}$, the external X-ray background is due solely to the DXRB. For a guard rate of $\sim 1000$, the count rate in the DXRB is approximately equal to the non-X-ray background count rate over $0.4-0.6 \mathrm{keV}$ and can be half the non-X-ray background count rate over $0.6-1.0 \mathrm{keV}$. However, the DXRB flux is less than $10 \%$ of the non-X-ray background at energies greater than $2 \mathrm{keV}$. The DXRB spectrum observed with BBXRT is presented by Jahoda et al. (1992) and will not be discussed further here. The diffuse background count rate remains roughly the same for all observations. This is because BBXRT is not particularly sensitive to spatial fluctuations in the DXRB due to short integration times.

\subsection{The Atmosphere and the X-Ray-Bright Earth}

The shuttle's orbit places it in Earth's upper atmosphere at an altitude of $\sim 200 \mathrm{~km}$. Because low-energy X-rays are absorbed and scattered at such altitudes, it is important to have an accurate understanding of the composition and ionization state of the atmosphere. At $200 \mathrm{~km}, \mathrm{~N}_{2}$ and $\mathrm{O}$ mainly constitute the atmosphere (Jones 1964; Mitra 1952; Zombeck 1990), and the ratio of $\mathrm{O}$ to $\mathrm{N}_{2}$ is approximately 1:1 (Zombeck 


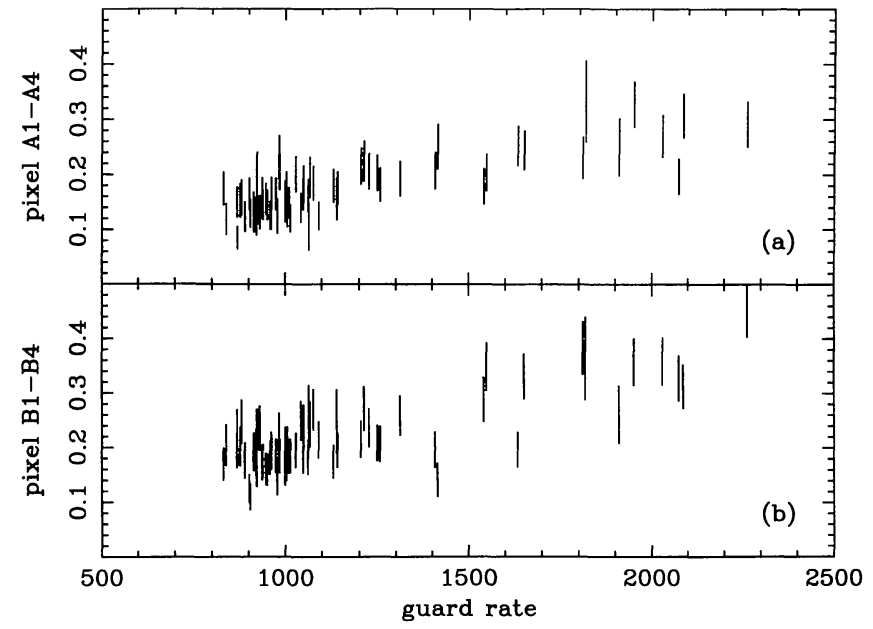

FIG. 21.-Non-X-ray background count rate vs. the A detector guard rate for $(a)$ all outer A pixels, channels $30-511$, and $(b)$ all outer B pixels, channels 30-511.

1990). In addition, the upper atmosphere below $700 \mathrm{~km}$ can be treated as neutral because at no time do the ions exceed their corresponding neutral species by more than $1 \%$ (Jones 1964).

BBXRT suffers from a large amount of low-energy (0.3-1.0 $\mathrm{keV}$ ) contamination during "day-side" observations. This contribution to the X-ray background occurs when X-ray photons from the solar corona are scattered by Earth's upper atmosphere. The relevant processes involved are Thomson scattering and fluorescent scattering. Thomson scattering dominates for energies greater than $\sim 0.6 \mathrm{keV}$. By modeling the radiative transfer of X-rays through the Earth's atmosphere, Snowden \& Freyberg (1993) and Fink, Schmitt, \& Harnden (1988) have determined that observed X-ray "bright Earth" light curves and spectra (from ROSAT and the Einstein IPC) can be predicted from a knowledge of the angle between the pointing direction of the telescope and the direction of Earth as well as the telescope-Earth-Sun angle.

BBXRT encountered two "states" of bright Earth emission: an "active" state when the Sun was flaring and a "quiet" state when the Sun was more or less quiescent. During the active state, when the Sun emits higher energy photons, emission lines of $\mathrm{Mg}, \mathrm{Si}$, and $\mathrm{Ar}$ are observed as well as a strong thermal continuum and $\mathrm{Fe} \mathrm{L}$ emission. Fortunately, no flares occurred during daytime source observations. During the quiet state, the BBXRT spectrum is dominated by a weaker thermal continuum and scattered Fe L emission as well as $\mathrm{O}$ and $\mathrm{N}_{2}$ fluorescence lines, and the observed intensity depends on the Earth angle. Spectra of the bright Earth for two ranges of Earth angles during the solar quiet state are shown in Figure 22. The Earth angle range of $70^{\circ}-80^{\circ}$ corresponds to looking directly at Earth's limb $\left(\sim 76^{\circ}\right.$ at $\left.200 \mathrm{~km}\right)$. Between $90^{\circ}$ and $100^{\circ}$, the spectrum is dominated by $\mathrm{O}$ fluorescence and is typical of what is seen during a daytime source observation. Figure 22 also illustrates how the Thomson-scattered continuum dies away for larger Earth angles.

We have investigated how the less than $2 \mathrm{keV}$ bright Earth count rate correlates with Earth angle. Figure 23 shows the

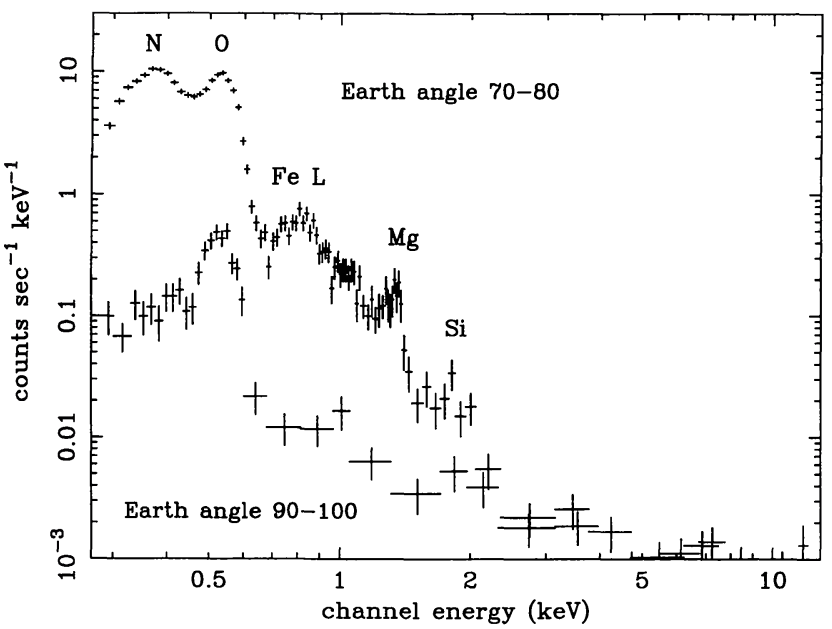

FIG. 22.-Quiescent solar scattered spectra for Earth angle ranges of $70^{\circ}-80^{\circ}$ and $90^{\circ}-100^{\circ}$. Data are from pixel A0.

change in count rate versus the Earth angle in three channel ranges: $27-42(0.5-0.6 \mathrm{keV}), 45-80(0.6-1.0 \mathrm{keV})$, and 80 $120(1.0-2.0 \mathrm{keV})$. The channel range $27-42$ emphasizes the oxygen line. The oxygen line is brightest at Earth's limb (between $70^{\circ}-80^{\circ}$ ), and there is a large drop in the count rate above $80^{\circ}$, although oxygen contamination persists up to $150^{\circ}$. Channels 45-80 emphasize the change in the $\mathrm{Fe} \mathrm{L}$ count rate with distance from Earth's limb. There is once again a rapid drop above $80^{\circ}$ with significant counts in the $\mathrm{Fe} \mathrm{L}$ complex even at an angle of $100^{\circ}$. For higher energies (channels 80 120 ) the same drop in count rate above $70^{\circ}-80^{\circ}$ occurs; for Earth angles larger than $80^{\circ}$, there is essentially no contribution to the background from energies greater than $1.2 \mathrm{keV}$. For Earth angles between $80^{\circ}$ and $100^{\circ}$, the best description of the

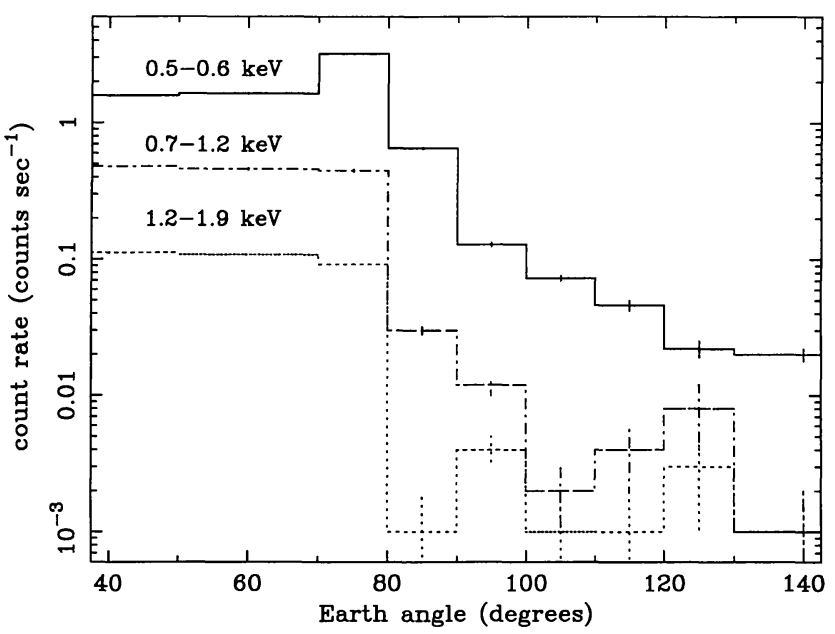

FIG. 23.-Change in daytime background count rate in pixel A2 with Earth angle for three channel ranges: channels $27-42(0.5-0.6 \mathrm{keV})$, channels $45-80(0.6-1.0 \mathrm{keV})$, and channels $80-120(1.0-2.0 \mathrm{keV})$. The non$\mathrm{X}$-ray background component has been subtracted. 
bright Earth spectrum is a Raymond-Smith plasma continuum with $k T \sim 0.43$ and $\sim 0.1$ solar abundance, along with nitrogen and oxygen fluorescence lines.

\section{BACKGROUND SUBTRACTION}

The non-X-ray component of the background can be easily predicted as follows. First, the average guard rate during the source observation (the "source guard rate" [SGR]) is determined (Table 1). Second, the count rate of the non-X-ray background, which has a mean guard rate (MGR) of 1259 , is scaled using the ratio of the MGR to the SGR to match the count rate that is predicted from the linear relation shown in Figure 21 . Finally, the scaled non-X-ray background is subtracted from the source data. The non-X-ray background reaches a lower threshold at a guard rate of 1000 and should not be scaled below this value. The background subtraction procedure discussed here only applies to observations out of the SAA with an average accumulated guard rate of $\lesssim 1400$.

At present, the nighttime diffuse background has only been examined for Earth angles greater than $90^{\circ}$. There is no evidence for oxygen fluorescence from the Earth's atmosphere above an Earth angle of $90^{\circ}$ in the BBXRT data. Creating an appropriate background for an observation obtained during orbit night consists of adding two background files together. These are (1) a DXRB spectrum which has its non-X-ray background already subtracted and (2) the scaled non-X-ray detector background as described above.

The bright Earth count rate remains constant for guard rates of 800-1800 within a given range of Earth angles, in contrast to the linear correlation seen between count rate and guard rate for the non-X-ray detector background. A combined "best" background spectrum for a daytime observation consists of (1) the daytime background (which contains scattered light plus the DXRB spectrum) accumulated for the average Earth angle of the source observation, minus its non-X-ray background, plus (2) the non-X-ray detector background that has been scaled as described above. These can be added together to form one daytime background spectrum.

The adopted background subtraction algorithm is given below. In this algorithm, $\mathrm{BG}_{N}$ is the nighttime (diffuse only) background, $\mathrm{BG}_{I}$ is the non-X-ray background, $\mathrm{BG}_{D}(\mathrm{EA})$ is the daytime (diffuse plus scattered) background as a function of Earth angle (usually given in degrees), NGR and DGR are the average guard rates of the nighttime and daytime backgrounds, respectively, and SGR is the average guard rate during the source observation.

BBXRT background subtraction algorithm: Orbit night:

Data $-\left[\mathrm{BG}_{N}-\mathrm{BG}_{I}\right.$ (scaled using NGR)]

$$
\text { - } \mathrm{BG}_{I} \text { (scaled using SGR); }
$$

Orbit day:

$$
\begin{array}{r}
\text { Data }-\left[\mathrm{BG}_{D}(\mathrm{EA})-\mathrm{BG}_{I}(\text { scaled using DGR })\right] \\
-\mathrm{BG}_{I}(\text { scaled using SGR }) .
\end{array}
$$

The fact that BBXRT has five detector elements makes the estimation of the simultaneous background fairly straightforward for weak point sources observed on axis. In this case, the simultaneous outer-pixel data provide a direct estimate of the background during the observation.

\section{REMAINING SYSTEMATIC UNCERTAINTIES}

We have presented the most up-to-date calibration of Goddard's BBXRT. We currently understand the effective area curve at the 5\%-10\% level over $0.7-10 \mathrm{keV}$ and at the $10 \%-$ $15 \%$ level below $0.7 \mathrm{keV}$ for data from a source pixel for both on- and off-axis observations of point sources. It is hoped that the techniques presented here are useful for the calibration of other present and future solid-state X-ray detectors. The calibration of the BBXRT detectors proved to be difficult because the problems encountered during the mission did not allow us to carry out our full calibration program. In fact, few adequate calibration targets were observed. Further refinements of the effective area will have to rely on postflight ground calibration measurements. A summary of the remaining calibration uncertainties and systematics, as fully explained in this paper, are listed below.

1. Our partial charge model does not properly account for the low energy $(<0.7 \mathrm{keV})$ shape of the partial charge distribution. This mainly affects the $\mathrm{B}$ detector and causes data from less than $0.6-0.7 \mathrm{keV}$ in $\mathrm{B} 1, \mathrm{~B} 2, \mathrm{~B} 3$, and $\mathrm{B} 4$ to be unusable.

2. A high-energy excess still exists in the B0 pixel above 9 $\mathrm{keV}$ ( an $\sim 10 \%$ effect). This is most likely due to incorrect modeling of the Ni edge at $8.3 \mathrm{keV}$.

3 . Low-energy residuals in the Crab data are seen. These may vary with time and are a 5\%-15\% effect below $0.6 \mathrm{keV}$.

4. Residuals still remain around the $\mathrm{Al}, \mathrm{Si}$, and $\mathrm{Au}$ edges due to a mismatch of our model to the Crab data (5\% effect).

5. Because we have not accounted for energy-dependent scattering out of the core of the mirror PSF, there is likely a systematic error for on-axis sources that causes the $2-10 \mathrm{keV}$ slopes in A0 and B0 to be too flat by about 5\%-10\%. This should only be significant for bright targets.

6. The systematic error in the measurement of the Crab slope between pairs of outer pixels (for source pixels) is \pm 0.10 in $\Gamma$.

7. The measured power-law indices for Crab spectra from nonsource pixels are too flat by $10 \%-25 \%$. This is a $\gtrsim 10 \%-20 \%$ effect in flux at greater than $5 \mathrm{keV}$ and may also result from not accounting for the energy-dependent mirror PSF.

This paper is the result of research toward the fulfillment of requirements of the Ph.D. degree at the University of Maryland. K. A. W. acknowledges the support of a NASA Graduate Researcher's Fellowship. We would like to acknowledge W. Focke for his assistance during postflight calibration data gathering. K. A. W. would also like to thank G. Banks for providing Figure 1 and G. Madejski for helpful discussions and a careful reading of the manuscript. 


\section{REFERENCES}

Agarwal, B. K. 1991, X-Ray Spectroscopy, (2d ed., New York: Springer) Bearden, J. A. 1967, Rev. Mod. Phys., 39, 78

Bearden, J. A., \& Burr, A. F. 1967, Rev. Mod. Phys., 39, 125

Blair, W. P., \& Gull, T. R. 1990, S\&T, 79, 591

Burrows, D. 1982, Ph.D. thesis, Univ. Wisconsin

Campbell, J. L., Millman, B. M., Maxwell, J. A., Perujo, A., \& Teesdale, W. J. 1985, Nucl. Instr. Meth. B, 9, 71

Fink, H. H., Schmitt, J. H. M. M., \& Harnden, F. R. 1988, A\&A, 193, 345

Fraser, G. W. 1989, X-ray Detectors in Astronomy (Cambridge: Cambridge Univ. Press)

Gould, R. J., \& Jung, Y. 1991, ApJ, 373, 271

Inagaki, Y., Shima, K., \& Maezawa, H. 1987, Nucl. Instr. Meth. B, 27, 353

Jahoda, K., et al. 1992, in The X-Ray Background, ed. X. Barcons \& A. C. Fabian (Cambridge: Cambridge Univ. Press), 240

Jones, L. M. 1964, in University of Michigan Engineering Summer Conferences on High Altitude Science (Ann Arbor: Univ. Michigan Press), $1: 2$

Joy, D. C. 1985, AT\&T Tech. Memo., TM 11521-850123-06

Krumrey, M., Tegeler, E., \& Ulm, G. 1988, Rev. Sci. Instr., 60, 2287

Lee, P. A., Citrin, P. H., Eisenberger, P., \& Kincaid, B. M. 1981, Rev. Mod. Phys., 53, 769

Mauche, C. W., \& Gorenstein, P. 1989, ApJ, 336, 843

Mitra, S. K. 1952, The Upper Atmosphere (2d ed.; Calcutta: Asiatic Soc.), 174
Morrison, R., \& McCammon, D. 1983, ApJ, 270, 119

Petre, R., \& Serlemitsos, P. J. 1985, Appl. Opt., 24, 1833

Petre, R., Serlemitsos, P. J., Marshall, F. E., Jahoda, K., \& Kunieda, H., 1992, Proc. SPIE, 1546, 72

Schattenburg, M. E., \& Canizares, C. R. 1986, ApJ, 301, 759

Serlemitsos, P. J. 1988, Appl. Opt., 27, 1447

Serlemitsos, P. J., Marshall, F. E., Petre, R., Jahoda, K., Boldt, E. A., Holt, S. S., Mushotzky, R., Swank, J., Szymkowiak, A., Kelley, R., \& Loewenstein, M. 1991, in Frontiers of X-ray Astronomy, ed. Y. Tanaka \& K. Koyama (Tokyo: Universal Academy Press), 221

Seward, F. 1992, Legacy, No. 2, 47

Shima, K., Nagai, S., Mikumo, T., \& Yasumi, S. 1983, Nucl. Instr. Meth., 217,515

Snowden, S. L., \& Freyberg, M. J. 1993, ApJ, 404, 403

Toor, A., \& Seward, F. D. 1974, AJ, 74, 995

Turner, M. J. L., et al. 1989, in Proc. 23d ESLAB Symp. on Two Topics in X-Ray Astronomy, ed. J. Hunt \& B. Battrick (ESA SP-296) (Noordwijk: ESA Publications Division ), 2, 769

Weaver, K. A. 1993, Ph.D. thesis, Univ. Maryland

Yacout, A. M., Gardner, R. P., \& Verghese, K. 1986, Nucl. Instr. Meth. A, 243,121

Zombeck, M. V. 1990, Handbook of Space Astronomy and Astrophysics (Cambridge: Cambridge Univ. Press), 239 